\title{
Galaxy Rotation Curves Without Non-Baryonic Dark Matter
}

\author{
J. R. Brownstein ${ }^{1}$ and J. W. Moffat ${ }^{2}$ \\ The Perimeter Institute for Theoretical Physics, Waterloo, Ontario, N2J 2W9, Canada \\ and \\ Department of Physics, University of Waterloo, Waterloo, Ontario N2Y 2L5, Canada
}

\begin{abstract}
We apply the modified acceleration law obtained from Einstein gravity coupled to a massive skew symmetric field $F_{\mu \nu \lambda}$ to the problem of explaining galaxy rotation curves without exotic dark matter. Our sample of galaxies includes low surface brightness (LSB) and high surface brightness (HSB) galaxies, and an elliptical galaxy. In those cases where photometric data are available, a best fit via the single parameter $(M / L)_{\text {stars }}$ to the luminosity of the gaseous (HI plus He) and luminous stellar disks is obtained. Additionally, a best fit to the rotation curves of galaxies is obtained in terms of a parametric mass distribution (independent of luminosity observations) - a two parameter fit to the total galactic mass, (or mass-to-light ratio $M / L$ ), and a core radius associated with a model of the galaxy cores using a nonlinear least-squares fitting routine including estimated errors. The fits are compared to those obtained using Milgrom's phenomenological MOND model and to the predictions of the Newtonian-Kepler acceleration law.
\end{abstract}

Subject headings: dark matter - galaxies: general — galaxies: kinematics and dynamics — galaxies: fundamental parameters

\section{Introduction}

In spite of intensive searches for the components of non-baryonic dark matter, no candidate particles have been observed. This leads one to search for a gravity theory that can

\footnotetext{
${ }^{1}$ jbrownstein@perimeterinstitute.ca

2 john.moffat@utoronto.ca
} 
explain galaxy dynamics without the need for exotic dark matter. Such a possible model was introduced in Milgrom (1983), and for more than twenty years since its publication, attempts to form a consistent relativistic theory containing Milgrom's phenomenological model have been pursued (Bekenstein 2004; Sanders 2005). A generalization of Einstein's general relativity (GR) based on a pseudo-Riemannian metric tensor and a skew symmetric rank three tensor field $F_{\mu \nu \lambda}$, called metric-skew-tensor-gravity (MSTG), leads to a modified acceleration law that can explain the flat rotation curves of galaxies and cluster lensing without postulating exotic dark matter (Moffat 2005a). A relativistic scalar-tensor-vector gravity (STVG) theory has also been developed which yields the same modified acceleration law as MSTG, and provides an effective description of the running of the $G, \gamma_{c}$ and $\mu$ with space and time (Moffat 2005b).

A cosmological model obtained from the field equations and a running of the effective gravitational coupling constant $G$ can also explain the growth of large scale structure formation without invoking cold dark matter. The running of the cosmological constant would produce a quintessence-like dark energy that could account for the acceleration of the expansion of the universe (Perlmutter et al. 1997; Riess et al. 1998, 2004; Garnavich et al. 1998; Spergel et al. 2003; Bennett et al. 2003).

A renormalization group (RG) framework (Reuter \& Weyer 2004a,b) for MSTG was developed to describe the running of the effective gravitational coupling constant $G$, and the effective coupling constant $\gamma_{c}$ that measures the strength of the coupling of the $F_{\mu \nu \lambda}$ field to matter. A momentum cutoff identification $k=k(x)$ associates the $\mathrm{RG}$ scales to points in spacetime. For the static, spherically symmetric solution, the RG flow equations allow a running with momentum $k$ and proper length $\ell(r)=1 / k$ and provides for a spatial variation of the effective Newton's coupling constant, $G=G(r)$; together with a variation of the skew field coupling constant, $\gamma_{c}(r)$, to matter; and the effective mass of the skew field $\mu=\mu(r)=$ $1 / r_{0}$ where $r$ denotes the radial coordinate. Such a "running" of coupling constants and mass are well-known from particle physics (e.g. quantum chromodynamics) and condensed matter and are applied here to the behavior of the infrared fixed point of the renormalization group method of quantum gravity. The form of $G(r)$ as a function of $r$, obtained from the modified Newtonian acceleration law, leads to agreement with solar system observations, terrestrial gravitational experiments and the binary pulsar PSR 1913+16 observations, while strong renormalization effects in the infrared regime at large distances lead to fits to galaxy rotation curves.

In this paper, a fit to both low surface brightness (LSB) and high surface brightness (HSB) galaxies over the range from dwarf galaxies to giant galaxies is achieved in terms of the total galaxy mass $M$ (or $M / L$ ) without exotic dark matter. A satisfactory fit is achieved 
to the rotational velocity data generic to the elliptical galaxy NGC 3379. A model of the modified acceleration law that includes a description of radial velocity curves in the core of galaxies as well as in the outer regions of the galaxy is shown to yield good fits to rotational velocity data. The significance of this result is made explicit by the depth of the galaxy rotation curve study, here. We performed photometric fits to 58 HSB and LSB and Dwarf galaxies utilizing a single parameter - the $M / L$ ratio of the stellar disk. 29 of these galaxies were based upon $B$-band and the remaining 29 galaxies were based upon $K$-band. The $K$-band data is the more modern data set and is a more precise tracer of the mean radial distribution of the dominant stellar population - these were the photometric results of Ursa Major. Since 2001, the Ursa Major data has been revised since the estimated distance to the cluster is now taken as 18.6 Mpc as opposed to the original publication's use of 15.5 Mpc. We updated the original analysis of Sanders \& Verheijen (1998) using that group's Groningen Image Processing System (GIPSY) ${ }^{1}$.

In addition, we performed 101 galaxy rotation curve fits to HSB and LSB and Dwarf galaxies (including the 58 galaxies that were fit photometrically) using a parametric mass distribution. These fits were necessary for those galaxies for which photometric data of the HI gas (corrected for He component) and stellar disk was unavailable. Although the model contains two parameters as opposed to one parameter for the photometric fits, it makes no assumptions on the constancy of the $M / L$ ratio within a galaxy although it does not distinguish between gas or stellar disk.

A comparison of the fits to the rotational velocity curve data obtained from MSTG and Milgrom's MOND reveals that the results from both models are qualitatively similar for almost all of the galaxy data fitted, although predictions of the galactic masses differ.

\section{Modified Acceleration Law}

From the derivation of the radial acceleration experienced by a test particle in a static, spherically symmetric gravitational field due to a point source, we obtain (Moffat 2005a,b):

$$
a(r)=-\frac{G_{\infty} M}{r^{2}}+\sigma \frac{\exp \left(-r / r_{0}\right)}{r^{2}}\left(1+\frac{r}{r_{0}}\right) .
$$

Here, $G_{\infty}$ is defined to be the effective gravitational constant at infinity

$$
G_{\infty}=G_{0}\left(1+\sqrt{\frac{M_{0}}{M}}\right),
$$

\footnotetext{
${ }^{1}$ http://www . astro.rug.nl/ gipsy/
} 
where $G_{0}$ is Newton's "bare" gravitational constant. This conforms with our definition of $G$ in the RG flow formalism, which requires that the effective $G$ be renormalized in order to guarantee that equation (1) reduces to the Newtonian acceleration

$$
a_{\text {Newton }}=-\frac{G_{0} M}{r^{2}}
$$

at small distances $r \ll r_{0}$. We model the coupling constant $\sigma$ for the repulsive Yukawa force contribution in equation (1) by

$$
\sigma=G_{0} \sqrt{M_{0} M}
$$

where $M_{0}$ is a coupling parameter. We obtain the acceleration on a point particle

$$
a(r)=-\frac{G_{\infty} M}{r^{2}}+G_{0} \sqrt{M M_{0}} \frac{\exp \left(-r / r_{0}\right)}{r^{2}}\left(1+\frac{r}{r_{0}}\right) .
$$

By using equation (2), we can express the modified acceleration in the form

$$
a(r)=-\frac{G_{0} M}{r^{2}}\left\{1+\sqrt{\frac{M_{0}}{M}}\left[1-\exp \left(-r / r_{0}\right)\left(1+\frac{r}{r_{0}}\right)\right]\right\} .
$$

We can rewrite equation (6) as

$$
a(r)=-\frac{G(r) M}{r^{2}}
$$

where

$$
G(r)=G_{0}\left\{1+\sqrt{\frac{M_{0}}{M}}\left[1-\exp \left(-r / r_{0}\right)\left(1+\frac{r}{r_{0}}\right)\right]\right\} .
$$

Thus, $G(r)$ describes the running with distance of the effective gravitational constant in the RG flow scenario.

The gravitational potential for a point source obtained from the modified acceleration formula (6) is given by

$$
\Phi(r)=\frac{G_{0} M}{r}\left[1+\sqrt{\frac{M_{0}}{M}}\left(1-\exp \left(-r / r_{0}\right)\right)\right]
$$

We apply equation (6) to explain the flatness of rotation curves of galaxies, as well as the approximate Tully-Fisher law (Tully \& Fisher 1977), $v^{4} \propto G_{0} M \propto L$, where $v$ is the rotational velocity of a galaxy, $M$ is the galaxy mass

$$
M=M_{\mathrm{stars}}+M_{H I}+M_{D B}+M_{f},
$$


and $L$ is the galaxy luminosity. Here, $M_{\text {stars }}, M_{H I}, M_{D B}$ and $M_{f}$ denote the visible mass, the mass of neutral hydrogen, possible dark baryon mass and gas, and the mass from the skew field energy density, respectively.

The rotational velocity of a star $v$ obtained from $v^{2}(r) / r=a(r)$ is given by

$$
v(r)=\sqrt{\frac{G_{0} M}{r}}\left\{1+\sqrt{\frac{M_{0}}{M}}\left[1-\exp \left(-r / r_{0}\right)\left(1+\frac{r}{r_{0}}\right)\right]\right\}^{1 / 2} .
$$

Let us postulate that the parameters $M_{0}$ and $r_{0}$ give the magnitude of the constant acceleration

$$
a_{0}=\frac{G_{0} M_{0}}{r_{0}^{2}} .
$$

We assume that for galaxies and clusters of galaxies this acceleration is determined by

$$
a_{0}=c H_{0}
$$

Here, $H_{0}$ is the current measured Hubble constant $H_{0}=100 h \mathrm{~km} \mathrm{~s}^{-1} \mathrm{Mpc}^{-1}$ where $h=$ $(0.71 \pm 0.07)$ (Eidelman et al. 2004) ${ }^{2}$. This gives

$$
a_{0}=6.90 \times 10^{-8} \mathrm{~cm} \mathrm{~s}^{-2} .
$$

We note that $a_{0}=c H_{0} \sim(\sqrt{\Lambda / 3}) c^{2}$, so there is an interesting connection between the parameters $M_{0}, r_{0}$ and the cosmological constant $\Lambda$.

Let us now describe a model of a spherically symmetric galaxy such that the density of visible matter, $\rho(r)$, contains an inner core at radius $r=r_{c}$. The acceleration law of equation (6) takes the form

$$
a(r)=-\frac{G_{0} \mathcal{M}(r)}{r^{2}}\left\{1+\sqrt{\frac{M_{0}}{M}}\left[1-\exp \left(-r / r_{0}\right)\left(1+\frac{r}{r_{0}}\right)\right]\right\},
$$

where

$$
\mathcal{M}(r)=4 \pi \int_{0}^{r} d r^{\prime} r^{\prime 2} \rho\left(r^{\prime}\right)
$$

is the total amount of ordinary matter within a sphere of radius $r$. A simple model for $\mathcal{M}(r)$ is

$$
\mathcal{M}(r)=M\left(\frac{r}{r_{c}+r}\right)^{3 \beta}
$$

\footnotetext{
${ }^{2}$ http://pdg. lbl.gov
} 
where

$$
\beta= \begin{cases}1 & \text { for HSB galaxies, } \\ 2 & \text { for LSB \& Dwarf galaxies }\end{cases}
$$

The constituents of $M$ in equation (15) and (17) are determined by equation (10). The density of ordinary matter is given by

$$
\frac{d \mathcal{M}(r)}{d r}=4 \pi r^{2} \rho(r) \equiv 3 \beta \mathcal{M}(r)\left[\frac{r_{c}}{r\left(r+r_{c}\right)}\right],
$$

so that we have

$$
\rho(r)=\frac{3}{4 \pi r^{3}} \beta \mathcal{M}(r)\left[\frac{r_{c}}{r+r_{c}}\right]
$$

Well inside the core radius, where $r \ll r_{c}$, the density $\rho(r) \propto$ constant for HSB galaxies with $\beta=1$. Although for LSB galaxies, it is the case for $\beta=2$ that $\rho(r) \propto\left(r / r_{c}\right)^{3}$ for $r \ll r_{c}$. This behavior is quickly suppressed and the density profile shows no rapid change for $r \ll r_{c}$. Indeed the choice of $\beta=2$ for LSB galaxies was found to be favorable because it resulted in a more slowly rising mass profile (the integrated density distribution) for small $r$. This critical distinction between high and low surface brightness galaxies has been studied in the context of cold dark matter, with the common conclusion that the rotation curves of LSB and Dwarf galaxies rise more slowly than those of HSB galaxies (or elliptical galaxies) (de Blok \& McGaugh 1996; de Blok et al. 2003; Bailin et al. 2005). However, the ordinary matter density of equation (20) does not exhibit the divergent or cuspy behavior for either HSB or LSB galaxies, contrary to the observations in these cold dark matter studies. Moreover, provided $r_{c} \ll r_{0}$ (which is the result for all the galaxies fitted), then we see that the exponential factor in the acceleration law of equation (15) has a damping effect well within the core radius such that the dynamics for $r \ll r_{c}$ is described by Newtonian theory. Thus the high resolution rotation curves for the LSB galaxies provide a clean testing ground for any theory of galaxy rotation curves. Without the distinction we have implemented by equation (18), the quality of our rotation curve fits would not be as good.

Well outside the core radius, where $r \gg r_{c}$, equation (17) implies that

$$
\lim _{r \gg r_{c}} \mathcal{M}(r)=M
$$

and the acceleration is described by equation (6).

For those galaxies for which there is photometric data available, the observed luminosity of the gaseous disk (HI plus He), and the stellar components (the disk and separately the bulge) can be used to provide a more precise fit to the velocity curve data, which would include the kinkiness of the rotation curves. By assuming a constant $M / L$ ratio, it is possible to invert the Poisson equation for the potential, yielding the mass distributions of the 
individual constituents. This results in a single parameter fit $-(M / L)_{\text {stars }}$. By including the bulge, a more precise fit is possible, in principle. However this would necessitate a second pa-

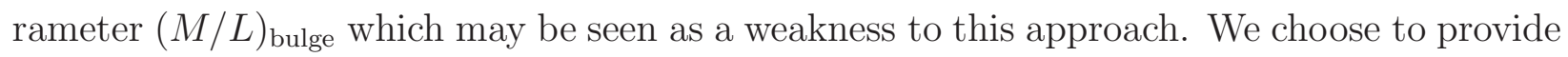
the single parameter fit - which we present for comparison. We note that the assumption of constant ratio $M / L$ forces an averaging over the radial distances thereby working to reduce the accuracy of the photometric fits. Our parametric mass model requires no assumptions on the ratio of $M / L$. The photometric fits also include the assumption that the HI gas is on stable circular orbits around the centers of the galaxies. This assumption may be violated for interacting galaxies, or in galaxies with strong bars. The photometric fits also utilize a correction of the mass for the Helium fraction, which is based on the results from Big-Bang nucleosynthesis and is not well known.

The rotational velocity derived from the acceleration law equation (15) is

$$
v(r)=\sqrt{\frac{G_{0} M}{r}}\left(\frac{r}{r_{c}+r}\right)^{\frac{3}{2} \beta}\left\{1+\sqrt{\frac{M_{0}}{M}}\left[1-\exp \left(-r / r_{0}\right)\left(1+\frac{r}{r_{0}}\right)\right]\right\}^{1 / 2} .
$$

The modified acceleration law equation (15) can be compared to the Newtonian law using equation (17):

$$
a_{\text {Newton }}(r)=-\frac{G_{0} \mathcal{M}(r)}{r^{2}}
$$

The gravitational acceleration described by Milgrom's phenomenological MOND model (Milgrom 1983; Sanders \& McGaugh 2002) is given by

$$
a \mu\left(\frac{a}{a_{0 \text { Milgrom }}}\right)=a_{\text {Newton }},
$$

where $\mu(x)$ is a function that interpolates between the Newtonian regime, $\mu(x)=1$, when $x \gg 1$ and the MOND regime, $\mu(x)=x$, when $x \ll 1$. The function normally used for galaxy fitting is

$$
\mu(x)=\frac{x}{\sqrt{1+x^{2}}} .
$$

\section{Galaxy Rotational Velocity Curves}

A good fit to LSB and HSB galaxy data is obtained with the parameters

$$
M_{0}=9.60 \times 10^{11} M_{\odot}, \quad r_{0}=13.92 \mathrm{kpc}=4.30 \times 10^{22} \mathrm{~cm},
$$

where we have substituted the value of $a_{0}$ from equation (14) into equation (12), relating the parameters $M_{0}$ and $r_{0}$. Thus, the modified acceleration law contains only a single parameter, 
$M_{0}$ or $r_{0}$, which once set as in equation (26) is universal for galaxies and is no longer a free parameter for fitting the galaxy rotation curves.

We allow for the smaller scale Dwarf galaxies, for which the outermost observed radial position, $r_{\text {out }} \lesssim 12 \mathrm{kpc}$, by rescaling equation (26) such that the values for $M_{0}$ and $r_{0}$ continue to satisfy the conditions (12) and (13):

$$
M_{0}=2.40 \times 10^{11} M_{\odot}, \quad r_{0}=6.96 \mathrm{kpc}=2.15 \times 10^{22} \mathrm{~cm} .
$$

The RG flow equations do indeed require that the parameters $M_{0}$ and $r_{0}$ are scale parameters and thus it is expected that while we may obtain reasonable fits assuming they are roughly constant within a set population, this is an approximation. We are able to fit all HSB galaxies with a fixed $M_{0}$ and $r_{0}$. We are also able to fit all LSB and Dwarf galaxies by rescaling the HSB fixed values of $M_{0}$ and $r_{0}$ by a factor of 4 and 2, respectively. This is done to keep the number of parameters to an absolute minimum while respecting the fact that the parameters $r_{0}$ and $M_{0}$ are determined by the scale of the system and should be different for HSB vs. LSB and Dwarf galaxies.

The fits to the galaxy rotation curves, $v$, in $\mathrm{km} / \mathrm{s}$ versus the galaxy radius, $r$, in $\mathrm{kpc}$ are shown in Figs. 1 through 5. The acceleration law is given by equation (15); and the rotational velocity by equation $(22)$.

\subsection{Photometric Velocity Curve Fits}

The fits to the data in Fig. 1 and 5 are based on the photometric data of the gaseous disk (HI plus He) component and luminous stellar disk component and only a single parameter $(M / L)_{\text {stars }}$ is used to fit the rotation curves. The data includes 29 galaxies both LSB and HSB galaxies - obtained from de Blok \& McGaugh (1998); de Blok et al. (2001a,b); Begeman et al. (1991); McGaugh et al. (2001); Sanders \& McGaugh (2002); Sanders (1996) and an additional 29 galaxies from the Ursa Major (UMa) cluster of galaxies derived from Sanders \& Verheijen (1998); Verheijen (2001); Verheijen \& Sancisi (2001). For the UMa cluster of galaxies, available $K$-band data was used to reproduce the velocity profiles of the gaseous disk (HI plus He) distribution and luminous stellar disks (via the ROTMOD task of GIPSY ${ }^{3}$ ). The gaseous disk (HI plus He) was assumed to be infinitely thin and to have a total mass given by

$$
M_{\text {gas }}=\frac{4}{3} M_{H I}
$$

\footnotetext{
${ }^{3}$ http://www. astro.rug.nl/ gipsy/
} 
where the He correction factor of $4 / 3$ is roughly determined from Big Bang nucleosynthesis, and the value of

$$
M_{H I}=2.36 \times 10^{5} D^{2} \int S d v\left[M_{\odot}\right],
$$

where $\int S d v$ is the integrated HI flux density in units of $\mathrm{Jy} \mathrm{km} / \mathrm{s}$ as measured from the global HI profile - taken from Column (15) of Table 2 of Verheijen (2001), and D is the distance in Mpc. We accounted for the revised distance estimate to UMa from $D=15.5 \mathrm{Mpc}$ to $D=18.6 \mathrm{Mpc}$ which has changed since the rotation curves were originally presented in Sanders \& Verheijen (1998). The luminous stellar disk was assumed to be described by the Van der Kruit and Searle law, where the disk density distribution as a function of $\mathrm{z}$ (vertical height from the plane of the disk) is given by

$$
\Sigma(z)=\operatorname{sech}^{2}\left(z / z_{0}\right) / z_{0},
$$

where $z_{0}$ is the vertical scale height of the luminous stellar disk, and was assumed to be $20 \%$ of the near infrared exponential disk scale length according to Column (13) of Table 2 of Verheijen (2001).

According to Sanders \& Verheijen (1998) the existence of $K$-band surface photometry is a great advantage since the near-infrared emission, being relatively free of the effects of dust absorption and less sensitive to recent star formation, is a more precise tracer of the mean radial distribution of the dominant stellar population. The principal advantages of using infrared luminosities is that stellar mass-to-light ratios are less affected by population differences and extinction corrections are minimal (Verheijen 2001). We focus on NGC 4010 in Fig. 5 to study the effect of the extended $\mathrm{HI}$ and $K$-band data (beyond the rotation curve data) on the quality fits in MSTG and MOND. The numerical results of the UMa fits are presented in Table 2.

\subsection{Parametric Velocity Curve Fits}

The fits to the rotation curve data shown in Figs. 2 through 4 are based on the parametric model of equations (17) and (18). Since this model is independent of the photometric data of the gaseous disk (HI plus He) component and luminous stellar disk component, a larger database of galaxies is available including the high resolution rotation curves of Sofue (1996) and the elliptical galaxy NGC 3379 of Romanowsky et al. (2003, 2004). This adds another 42 galaxies to the complete sample as described in Table 1. We focus on the Milky Way in Fig. 3 and NGC 3379 in Fig. 4 to clarify the predictions of MOND and MSTG which are hard to distinguish in Fig. 2; but become apparent at distances beyond the edge of the visible stars. The numerical results of the fits to the complete sample are presented in Table 3. 


\subsection{Flat Rotation Velocity}

In Milgrom's phenomenological MOND model we have

$$
v_{c}^{4}=G_{0} M a_{0 \text { Milgrom }}
$$

We see that equation (31) predicts that the rotational velocity is constant out to an infinite range and the rotational velocity does not depend on a distance scale, but on the magnitude of the acceleration $a_{0 \text { Milgrom }}$. In contrast, our modified acceleration formula does depend on the radius $r$ and the distance scale $r_{0}$, which for galaxies is fixed by the formula equation (13). The MSTG velocity curve asymptotically becomes the same as the Newtonian-Kepler prediction as $r \rightarrow \infty$ :

$$
v \sim \sqrt{G_{\infty} M / r}
$$

where $G_{\infty}$ is the renormalized value of Newton's constant.

The flatness of the rotation curves arises due to an increased strength in the galactic gravitational potential due to the running of Newton's constant. By taking the first and second derivative of $G(r)$ from equation (8):

$$
\begin{aligned}
\frac{d G(r)}{d r} & =\frac{G_{0}}{r_{0}^{2}} \sqrt{\frac{M_{0}}{M}} r \exp \left(-r / r_{0}\right), \\
\frac{d^{2} G(r)}{d r^{2}} & =\frac{G_{0}}{r_{0}^{2}} \sqrt{\frac{M_{0}}{M}}\left(1-\frac{r}{r_{0}}\right) \exp \left(-r / r_{0}\right),
\end{aligned}
$$

and we see that $d G(r) / d r$ has a maximum at $r=r_{0}$. Therefore the rate of change of the running of the effective gravitational constant has a maximum; and it is at this point where the tendency to return to the Newtonian-Kepler behaviour of equation (32) is most opposed by the RG flow. The effect of this on the galaxy rotation curves is to produce an extended region where the curve seems flat. The velocity at $r=r_{0}$ is defined as $v_{0}$ :

$$
v_{0} \equiv v\left(r=r_{0}\right)
$$

is the MSTG equivalent of the flat rotational velocity; and is within the quoted experimental uncertainties to the MOND equivalent, $v_{c}$, in those galaxies that MOND handles well. The numerical results for $v_{0}$ and $v_{c}$ with calculated uncertainties are included in Tables 2 and 3.

Using the Sloan Digital Sky Survey (SDSS), Prada et al. (2003) have studied the velocities of satellites orbiting isolated galaxies. They detected approximately 3000 satellites, and they found that the line-of-sight velocity dispersion of satellites declines with distance to the primary. The velocity was observed to decline to a distance of $\sim 350 \mathrm{kpc}$ for the available data. This result contradicts the constant velocity prediction equation (31) of MOND, but 
is consistent with the MSTG prediction equation (32). It also agrees with the cosmological models which predict mass profiles of dark matter halos at large distances. During the last two decades of numerical modelling of galaxy formation, they have produced a density profile of dark matter halos, $\rho \propto 1 / r^{3}$ at large radii, which does not depend on the nature of the dark matter (Avila-Reese et al. 2001; Colin et al. 2002). The results of Prada et al. (2003) are consistent with recent gravitational lensing results (Sheldon et al. 2003).

\section{The Tully-Fisher Relation}

Unlike MOND, the mass - rotational velocity relationship is not absolute as in equation (31); and indeed at distances beyond the galaxy it is expected that Kepler's Law applies according to equation (32).

The observational Tully-Fisher relation implies a luminosity - rotational velocity of the form (Tully \& Fisher 1977):

$$
L \propto v_{\text {out }}^{a} \text { where } a \approx 4,
$$

where $L$ is the total observed luminosity of the galaxy (in units of $10^{10} L_{\odot}$ ), and $v_{\text {out }}$ is the observed velocity at the outermost observed radial position (in $\mathrm{km} / \mathrm{s}$ ). Verheijen (2001) considers an alternate definition of the "flat rotation velocity", $v_{\text {flat }}$, which categorizes galaxies according to three kinds depending on the shape of the rotation curve. The behavior of $v_{\text {out }}$ is more closely related to the asymptotic "flat rotation velocity". Taking the logarithm of both sides of equation (36), we obtain

$$
\log (L)=a \log \left(v_{\text {out }}\right)+b
$$

$B$-band luminosity data is available for practically all of the galaxies either from the original references listed in Table 3 or listed in Tully (1988). Moreover, the majority of the galaxies in this study have been detected by 2MASS in the $K_{s}$-band. In order to calculate the total $K$-band luminosity, apparent $K$-band magnitudes from the 2MASS surver were used. Given an apparent $K$-band magnitude it is possible to calculate the $K$-band luminosity as

$$
\log _{10}\left(L_{K}\right)=1.364-\frac{2}{5} K_{T}+\log _{10}(1+z)+2 \log _{10} D
$$

where $L_{K}$ is the $K$-band luminosity in units of $10^{10} L_{\odot}, K_{T}$ is the $K$-band apparent magnitude and $\mathrm{z}$ is the redshift of the galaxy (determined from the NASA/IPAC Extragalactic Database), and D is the distance to the galaxy in Mpc (from the original references). The $\log _{10}(1+z)$ term is a first order $K$-correction. We have plotted the observed Tully-Fisher relation for LSB and HSB galaxies in Fig. 6. 
As in (Sanders \& Verheijen 1998), MOND is able to make predictions on both the slope and the intercept of the logarithmic Tully-Fisher relation by assuming that the mass to luminosity ratio, $M / L$, is constant across all galaxies. Although this is not the case, the assumption is enforced by using the mean mass to light ratio, $\langle M / L\rangle$, to determine the values of the slope and intercept, $a$ and $b$, in equation (37). Replacing the observed luminosity, $L$, in the logarithmic Tully-Fisher relation of equation (37) with

$$
L=\frac{M}{\langle M / L\rangle}
$$

We obtain

$$
\log (M)=a \log \left(v_{\text {out }}\right)+b-\log (\langle M / L\rangle) .
$$

Thus, the effect of $\langle M / L\rangle$ is to shift the intercept which vanishes when $\langle M / L\rangle=1$. We may quantify the predictions of MSTG and MOND by either computing the appropriate $\langle M / L\rangle$ values which depend on the particular band of the luminosity measurements, or by considering the respective curve fits to the actual Tully-Fisher relation:

$$
\begin{array}{ll}
\log (M)=a \log \left(v_{0}\right)+b & \text { MSTG } \\
\log (M)=a \log \left(v_{c}\right)+b & \text { MOND }
\end{array}
$$

where $v_{0}$ is the MSTG "flat rotation velocity" of equation (35); and $v_{c}$ is the MOND asymptotic rotation velocity of equation (31). Taking the logarithm (with respect to base 10) of both sides of equation (31), we have the MOND predictions

$$
\begin{aligned}
a & =4 \\
b & =-8.21
\end{aligned}
$$

Fig. 7 shows the curve fits to equations (41) and (42) for both the photometric fits and the fits to our parametric model of equations (17) and (18). In all cases, we are able to combine the HSB, LSB and Dwarf galaxy data (and the elliptical galaxy NGC 3379) for the fitting, implying consistent physics across galaxies. The numerical results of the respective fits are presented in Table 4.

We see that a comparison of the MSTG and MOND results of the actual and observed Tully-Fisher relation show that the MSTG prediction is closer to the observational data for the complete sample and the UMa $B$-band photometry. This is most likely the result of the implicit assumption within the MOND framework that $\mathrm{M} / \mathrm{L}$ is constant within each galaxy which does not appear to be the case in either the $B-$ or $K-$ bands. 


\section{Conclusions}

A gravity theory consisting of a metric-skew-tensor action that leads to a modified Newtonian acceleration law (Moffat 2005a,b) can be fitted to a large class of galaxy rotation curves. We have presented the predictions for the galaxy rotation curves from a covariant and relativistic gravitational theory without postulating non-baryonic dark matter. The only other relativistic gravity theories that have been published that have attempted to do this are Mannheim (1990, 2005) and Bekenstein (2004). The latter recent publication attempts to incorporate Milgrom's MOND into a covariant gravitational theory. The fully relativistic gravitational theory presented in Moffat (2005a,b) is a consistent and viable example of a gravitational theory that fits the galaxy rotation curves and galaxy cluster mass X-ray data without non-baryonic dark matter Brownstein \& Moffat (2005). The large sample of galaxy data which fits our predicted MSTG acceleration law warrants taking seriously the proposal that a modified gravity theory can explain the flat rotational velocity curves of galaxies without (as yet, undetected) non-baryonic dark matter. It represents an important foil in comparing modified gravitational theory with dark matter.

It is interesting to note that we can fit the rotational velocity data of galaxies in the distance range $0.02 \mathrm{kpc}<r<70 \mathrm{kpc}$ and in the mass range $10^{5} M_{\odot}<M<10^{11} M_{\odot}$ without exotic dark matter halos. The lensing of clusters can also be explained by the theory without exotic dark matter in cluster halos. An important prediction is that for large enough distances from the galaxy cores, the rotational velocity of stars declines as $v \propto 1 / \sqrt{r}$ consistent with a Newtonian-Kepler fall off. This is consistent with the results of Prada et al. (2003) and gravitational lensing results for galaxies clusters (Sheldon et al. 2003).

In order to obtain a self-consistent description of solar dynamics, galaxies, clusters of galaxies and cosmology, it is necessary to have the effective gravitational constant $G$, the MSTG coupling constant $\gamma_{c}$, the mass $\mu$ (range $r_{0}$ ) of the skew field $F_{\mu \nu \lambda}$ and the cosmological constant $\Lambda$ run with distance (time). The RG flow effective action description of MSTG quantum gravity allows for a running of these effective constants with distance and time (Moffat 2005a,b). The RG flow framework for the theory is characterized by special $R G$ trajectories. On the RG trajectory, we identify a regime of distance scales where solar system gravitational physics is well described by GR, which is contained in MSTG as an approximate solution to the field equations. We are able to obtain agreement with the observations in the solar system, terrestrial gravitational experiments and the binary pulsar PSR 1913+16. Strong infrared renormalization effects become visible at the scale of galaxies and the modified Newtonian potential replaces exotic dark matter as an explanation of flat rotation curves. Thus, gravity becomes a "confining force" that has significant predictions 
for astrophysics and cosmology.

We have demonstrated that the RG flow running of $G$ and MSTG cosmology can lead to a description of the universe that does not require dominant, exotic dark matter. Dark energy is described by an effective time dependent cosmological constant. A detailed investigation of the MSTG cosmological scenario must be performed to establish that it can describe the large scale structure of the universe, account for galaxy formation and big bang nucleosynthesis and be consistent with the WMAP data.

The predictions for the galaxy rotation curves from MSTG and Milgrom's MOND agree remarkably for almost all of the 101 galaxies fitted throughout the visible distance scales of the galaxies for LSBs as well as HSBs and the one elliptical galaxy NGC 3379. In particular, for the fits using photometric data and only one parameter $\langle M / L\rangle$ (once $r_{0}$ and $M_{0}$ are fixed), the agreement of the two models suggests that Milgrom's MOND critical acceleration

$a_{0 \text { Milgrom }}=1.2 \times 10^{-8} \mathrm{~cm} / \mathrm{s}^{2}$ is closely related to the range parameter $r_{0}$ and the distance scaling behavior of the MSTG modified acceleration law.

\section{Acknowledgments}

This work was supported by the Natural Sciences and Engineering Research Council of Canada. JRB would like to thank the Perimeter Institute for Theoretical Physics for additional funding. We thank Martin Green and Martin Reuter for helpful discussions. We also thank Stacy McGaugh and Marc Verheijen for supplying data and for helpful discussions; and Erwin de Blok for assisting in our deployment of the Groningen Image Processing System $(\text { GIPSY })^{4}$ and the ROTMOD task on the Mac G4 platform.

\section{REFERENCES}

Avila-Rees, V., Colin, P., Valenzuela, O., D’Onghia, E. \& Firmani, C. 2001, ApJ, 559, 516 (astro-ph/0010525)

Bailin, J., Power, C., Gibson, B.K. \& Steinmetz M. 2005, MNRAS, submitted (astro-ph/0502231)

Bekenstein, J. D. 2004, Phys. Rev. D70, 083509 (astro-ph/0403694)

\footnotetext{
${ }^{4}$ http://www. astro.rug.nl/ gipsy/
} 
Begeman, K. G., Broeils, A.H. \& Sanders, R.H. 1991 MNRAS, 249, 523 (1991MNRAS . 249 . 523B)

Bennett, C. L. et al. 2003, ApJS, 148, 1 (astro-ph/0302207)

Brownstein, J. R. \& Moffat, J. W. 2005, preprint (astro-ph/0507222)

Colin, P., Avila-Rees, V., Valenzuela, O. \& Firmani, C. 2002, ApJ, 581, 777 (astro-ph/0205322)

de Blok, W. J. G. \& McGaugh, S. S. 1996, ApJ, 469, 89 (astro-ph/9607042)

de Blok, W. J. G. \& McGaugh, S. S. 1998, ApJ, 508, 132 (astro-ph/9805120)

de Blok, W. J. G., McGaugh, S.S., Bosma, A. \& Rubin, V. C. 2001, ApJ, 552, L23 (astro-ph/0103102)

de Blok, W. J. G., McGaugh, S. S. \& Rubin, V. C. 2001, AJ, 122, 2396 (astro-ph/0107366)

de Blok, W. J. G., Bosma, A. \& McGaugh, S. S. 2003, MNRAS, 340, 657 (astro-ph/0212102)

Eidelman, S. et al. 2004, Phys. Lett. B592, 1

Garnavich, P. M. et al. 1998, ApJ, 509, 74 (astro-ph/9806396)

McGaugh, A., Rubin, V.C. \& de Blok, W. J. G. 2001, AJ, 122, 2381 (astro-ph/0107326)

Mannheim, P.D. 2005, preprint (astro-ph/0505266)

Mannheim, P. D. 1990, Gen. Rel. Grav., 22, 289

Milgrom, M. 1983, ApJ, 270, 365

Moffat, J. W. 2005, J. Cosmology Astropart. Phys., 05, 003 (astro-ph/0412195)

Moffat, J. W. 2005, preprint (gr-qc/0506021)

Perlmutter, S. et al. 1997, ApJ, 483, 565 (astro-ph/9608192)

Prada, F. et al. 2003, ApJ, 598, 260 (astro-ph/0301360)

Riess, A. G. et al. 1998, AJ, 113, 1009 (astro-ph/9805201)

Riess, A. G. et al. 2004, ApJ, 607, 665 (astro-ph/0402512)

Reuter, M. \& Weyer, H. 2004, J. Cosmology Astropart. Phys., 12, 001 (hep-th/0410119) 
Reuter, M. \& Weyer, H. 2004, Phys. Rev. D69, 104022 (hep-th/0311196)

Romanowsky, A.J., Douglas, N.D., Arnaboldi, M., Kuijken, K, Merrifield, M.R., Napolitano, N.R., Capaccioli \& M., Freeman, K. C. 2003, Science, 301, 1696 (astro-ph/0308518)

Romanowsky, A. J. et al. 2004, in Proc. IAU Symposium No.220, Dark matter in Galaxies, ed. S. Ryder, D. J. Pisano, M. Walker \& K. Freedman, (San Francisco: ASP) 165 (astro-ph/0310874)

Sanders, R. H. 1996, ApJ, 473, 117 (astro-ph/9606089)

Sanders, R. H. 2005, preprint (astro-ph/0502222)

Sanders, R. H. \& McGaugh, S. S. 2002, ARA\&A40, 263 (astro-ph/0204521)

Sanders, R.H. \& Verheijen, M. A. W. 1998, ApJ, 503, 97 (astro-ph/9802240)

Shapiro, I. L., Sola, J. \& Stefancic, H. 2005, J. Cosmology Astropart. Phys., 01, 012 (hep-ph/0410095)

Sheldon, E. S. et al. 2003, AJ, 127, 2544 (astro-ph/0312036)

Sofue, Y. 1996, ApJ, 458, 120 (astro-ph/9507098)

Spergel, D. N. et al. 2003, ApJS, 148, 175 (astro-ph/0302209)

Tully, R. B. 1988, Nearby Galaxies Catalog (Cambridge, Cambridge University Press)

Tully, R. B. \& Fisher, J. R. 1977, A\&A, 54, 661

Verheijen, M. A. W. 2001, ApJ, 563, 694 (astro-ph/0108225)

Verheijen, M. A. W. \& Sancisi, R. 2001, A\&A, 370, 765 (astro-ph/0101404)

Submitted to ApJ, June 20, 2005. Accepted for publication in ApJ, September 21, 2005.

Journal Ref: ApJ 636 (January 10, 2006). 
Fig. 1.- Photometric Fits to Galaxy Rotation Curves: There are 58 galaxies presented here, each is a best fit via the single parameter $(M / L)_{\text {stars }}$ based on the photometric data of the gaseous (HI plus He) and luminous stellar disks. The 29 galaxies labeled UMa are members of the Ursa Major cluster of galaxies. For the UMa subset, available $K$-band data was used to reproduce the velocity profiles of the gaseous disk (HI plus He) distribution and luminous stellar disks accounting for the revised distance estimate to UMa from $15.5 \mathrm{Mpc}$ to 18.6 Mpc according to Verheijen \& Sancisi (2001). The numerical results of the UMa fits are presented in Table 2. In all cases, the radial coordinate (horizontal axis) is given in $\mathrm{kpc}$ and the rotation velocity (vertical axis) in $\mathrm{km} / \mathrm{s}$. The red points with error bars are the observations, the solid black line is the rotation curve determined from MSTG, the dash-dot cyan line is the rotation curve determined from MOND. The other curves are the Newtonian rotation curves of the various separate components: the long dashed green line is the rotation curve of the gaseous disk (HI plus He); the dotted magenta curve is that of the luminous stellar disk.

Fig. 2.- Parametric Fits to Galaxy Rotation Curves: There are 101 galaxies presented here - the complete sample of galaxies of Table 1 . These rotation curves are best fit to the parametric mass distribution (independent of luminosity observations) of equations (17) and (18) - a two parameter fit to the total galactic Mass, $M$, and a core radius, $r_{c}$. In all cases, the red points (with error bars) are the observations. In those cases where the high resolution observations connect together, error bars if available are shown as region specific. The solid black line is the rotation curve determined from MSTG, the dash-dot cyan line is the rotation curve determined from MOND. The horizontal dotted black line is the MSTG predicted value of the measured "flat rotation velocity", $v_{0}$ of equation (35). The remaining curve - the short dashed blue line is the Newtonian galaxy rotation curve. The numerical results of the fits are presented in Table 3. 

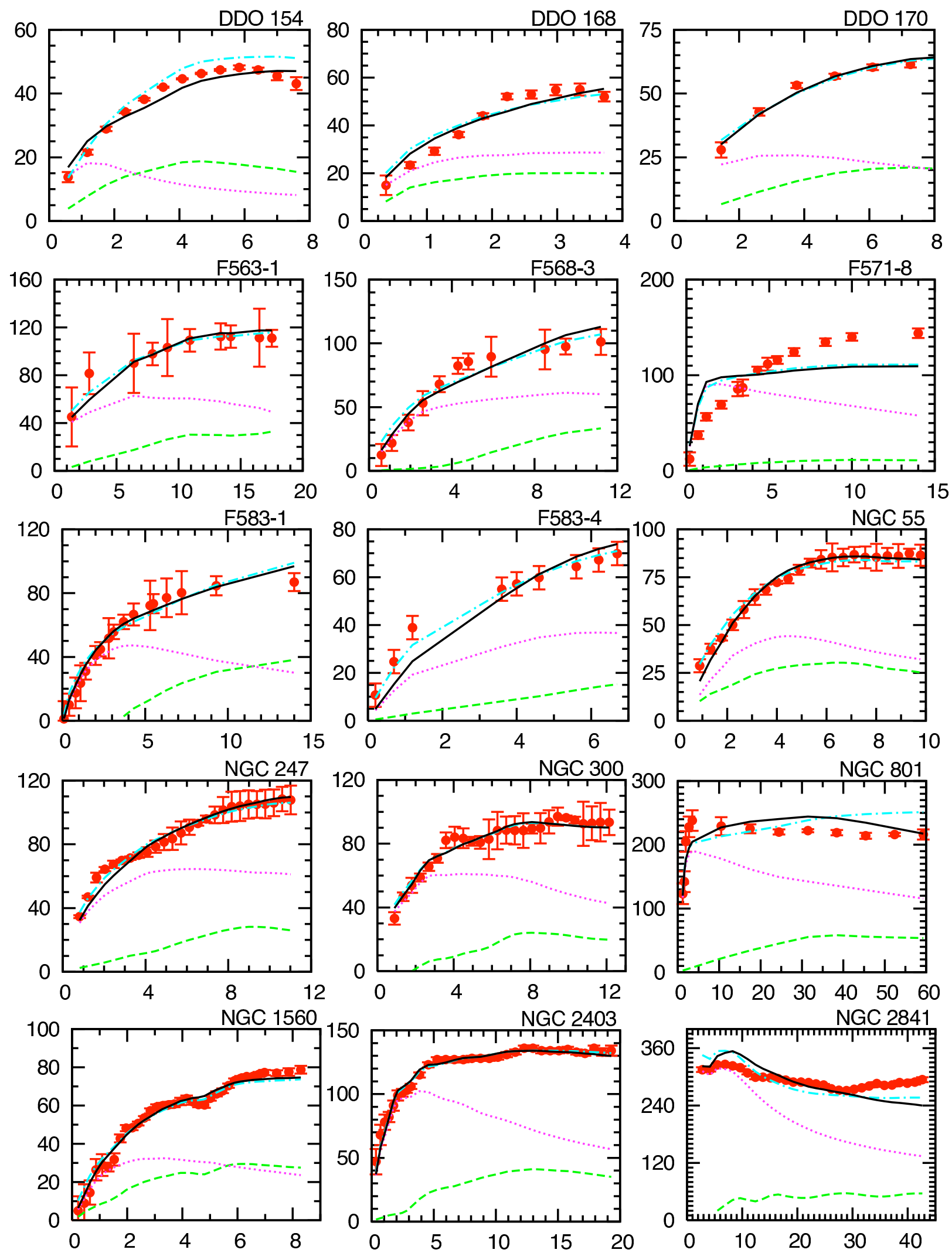

Fig. 1.- Photometric Galaxy Rotation Curve Fits 

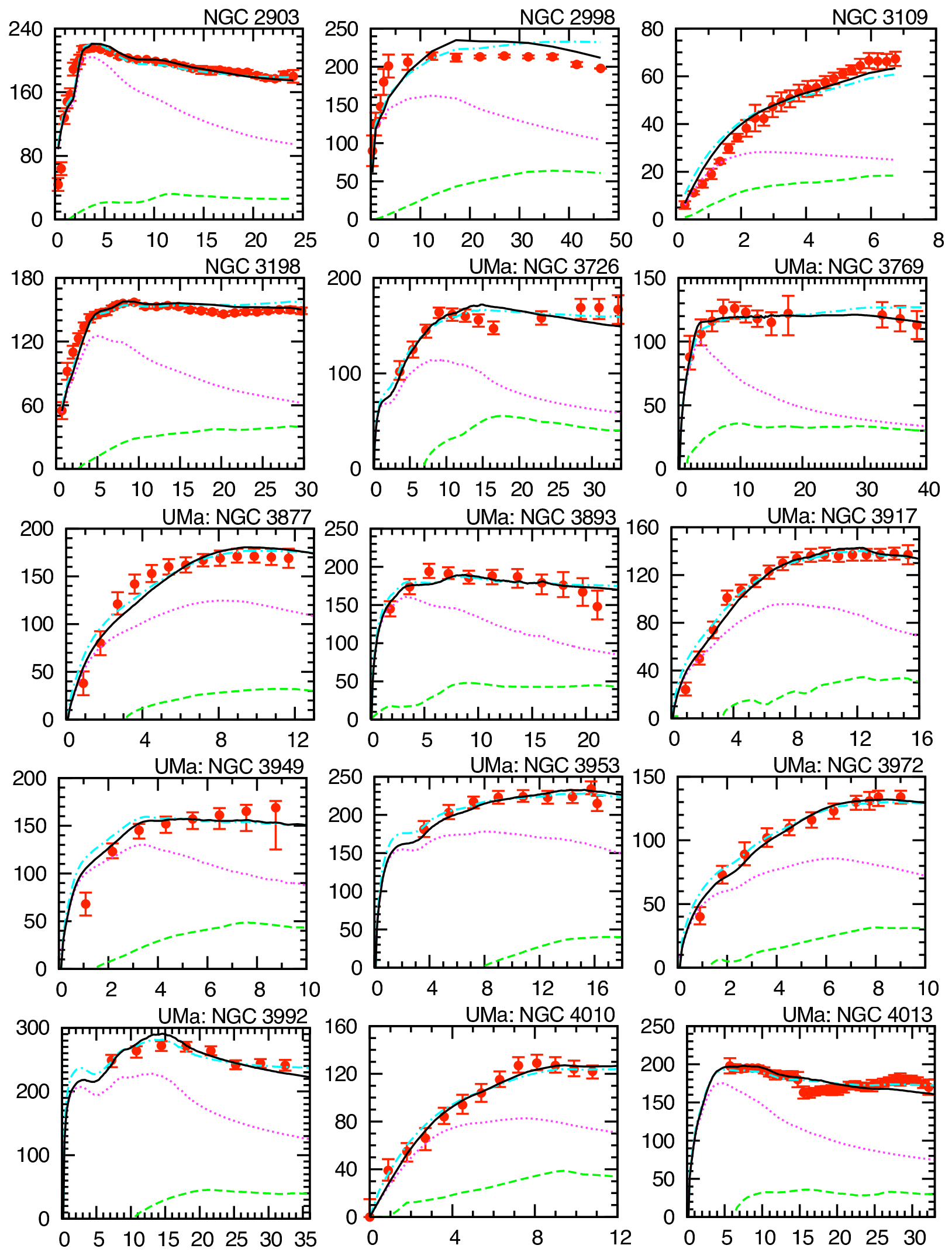

Fig. 1 Continued.- Photometric Galaxy Rotation Curve Fits 

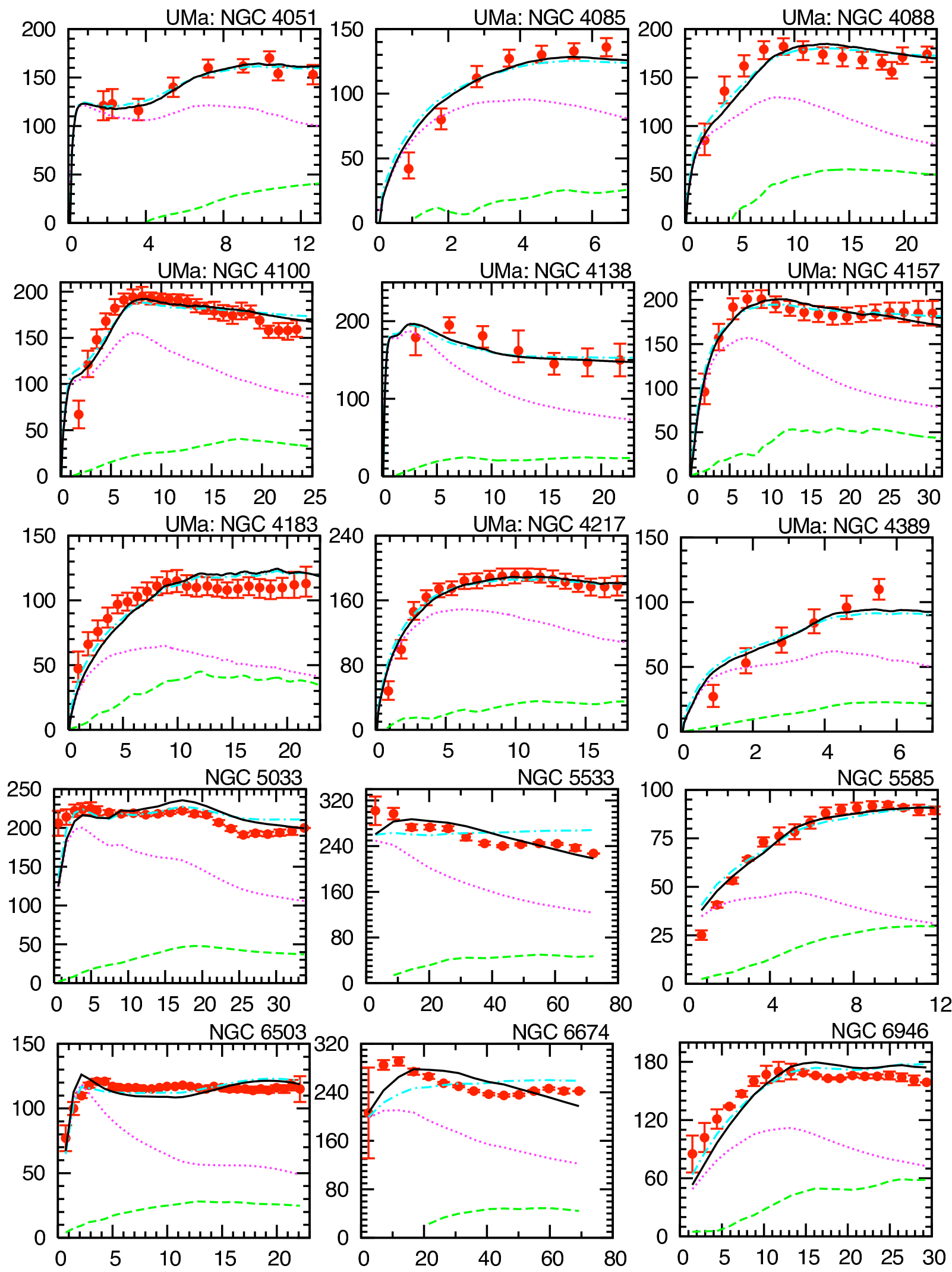

Fig. 1 Continued.- Photometric Galaxy Rotation Curve Fits 

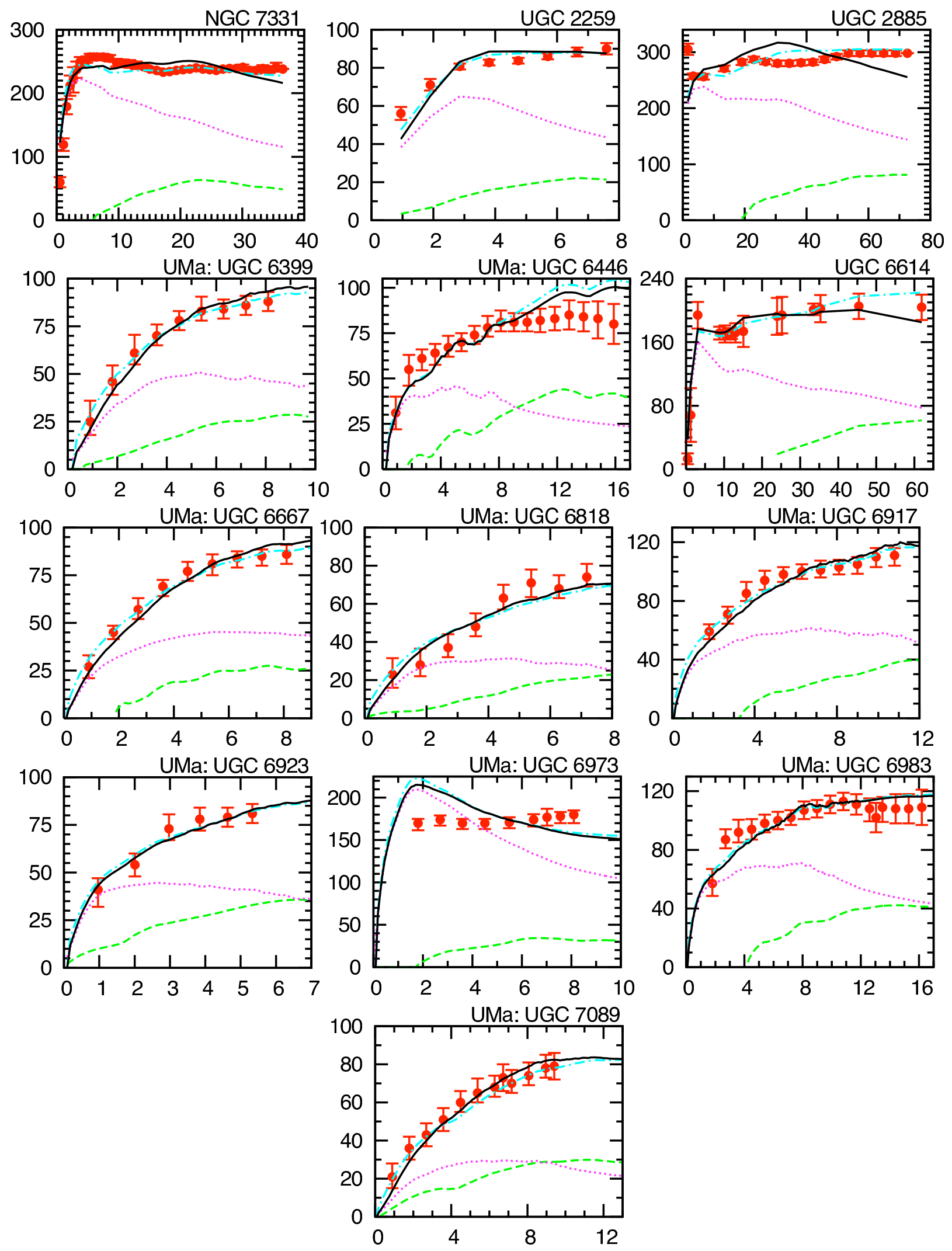

Fig. 1 Continued.- Photometric Galaxy Rotation Curve Fits 

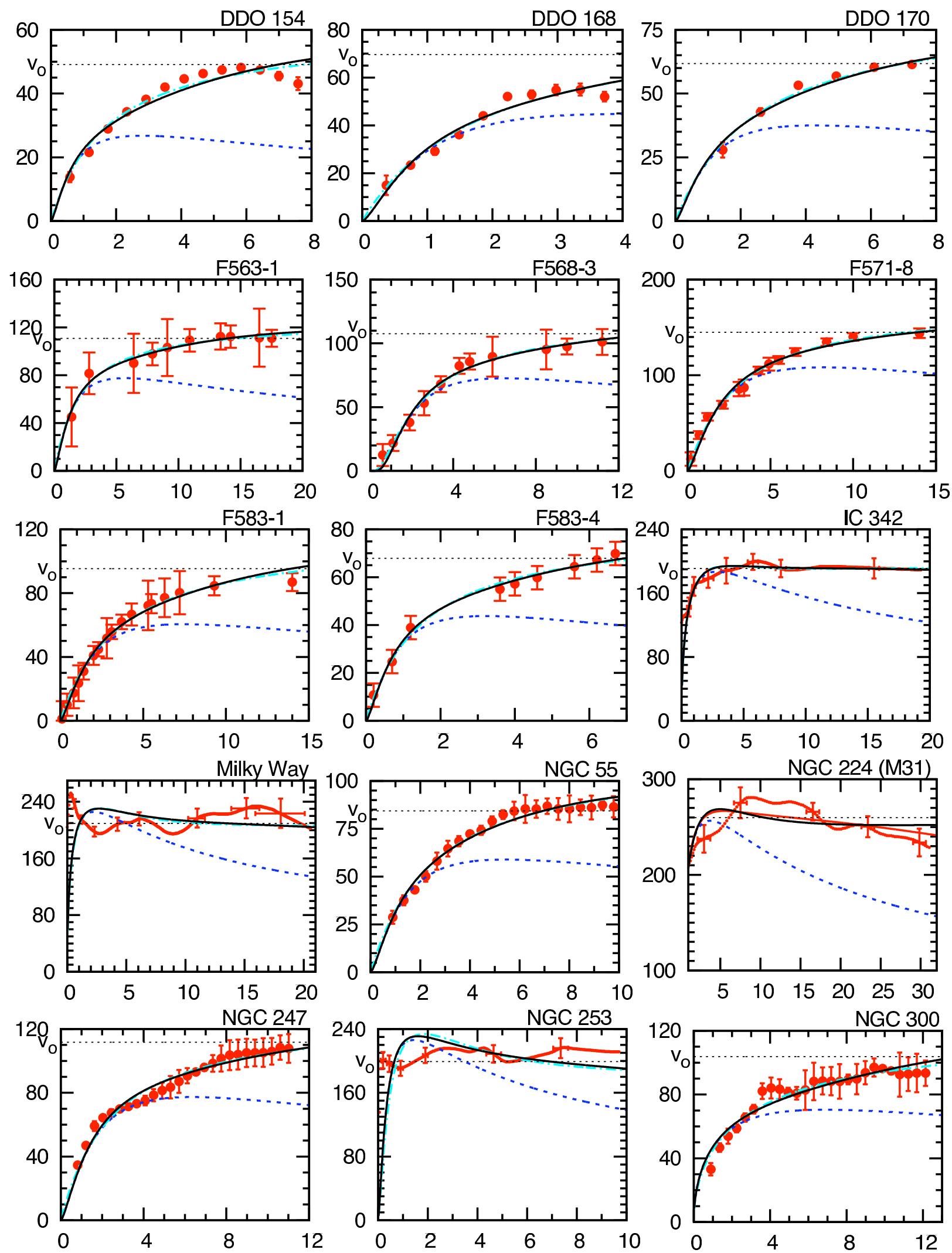

Fig. 2.- Parametric Galaxy Rotation Curve Fits 

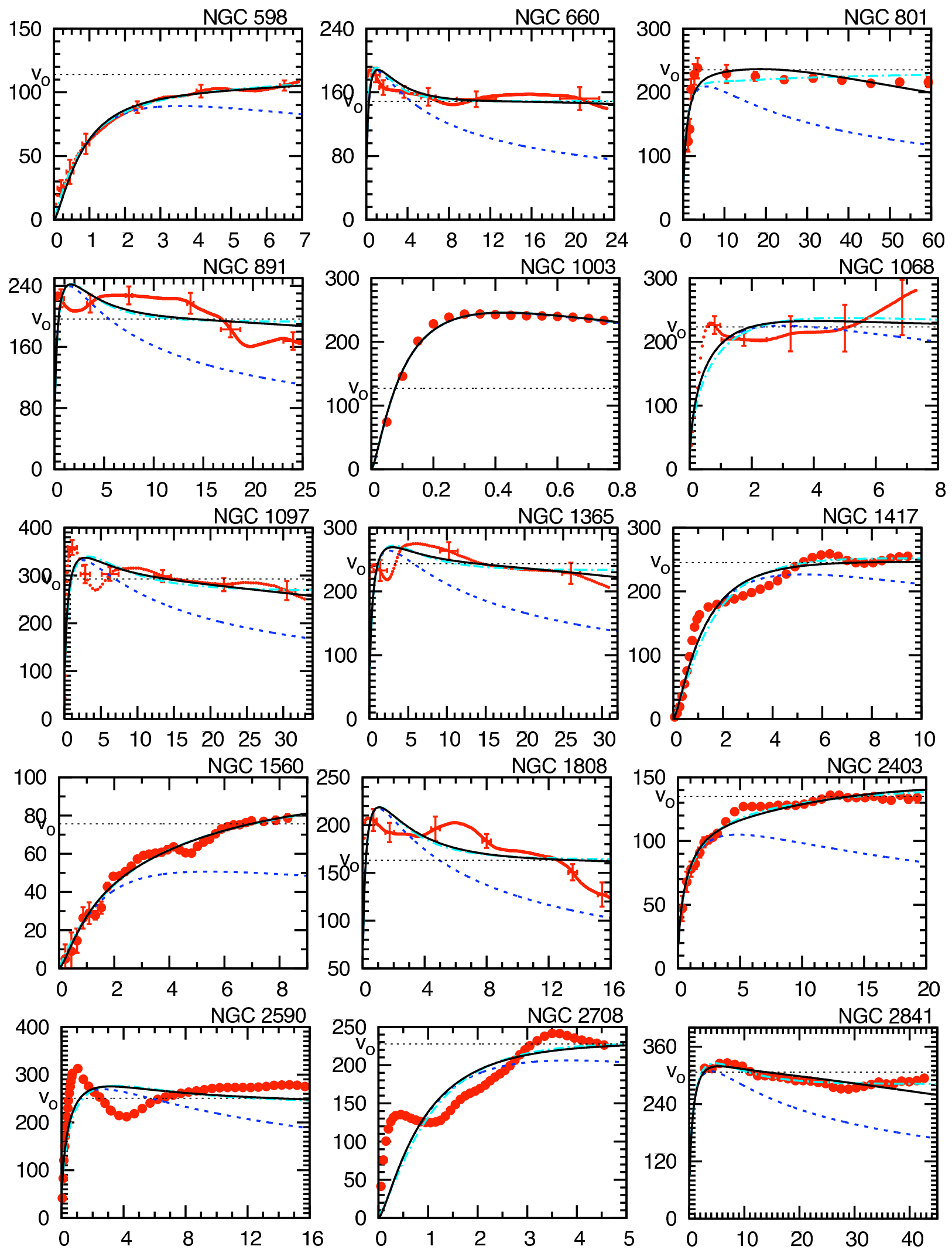

Fig. 2 Continued.- Parametric Galaxy Rotation Curve Fits 

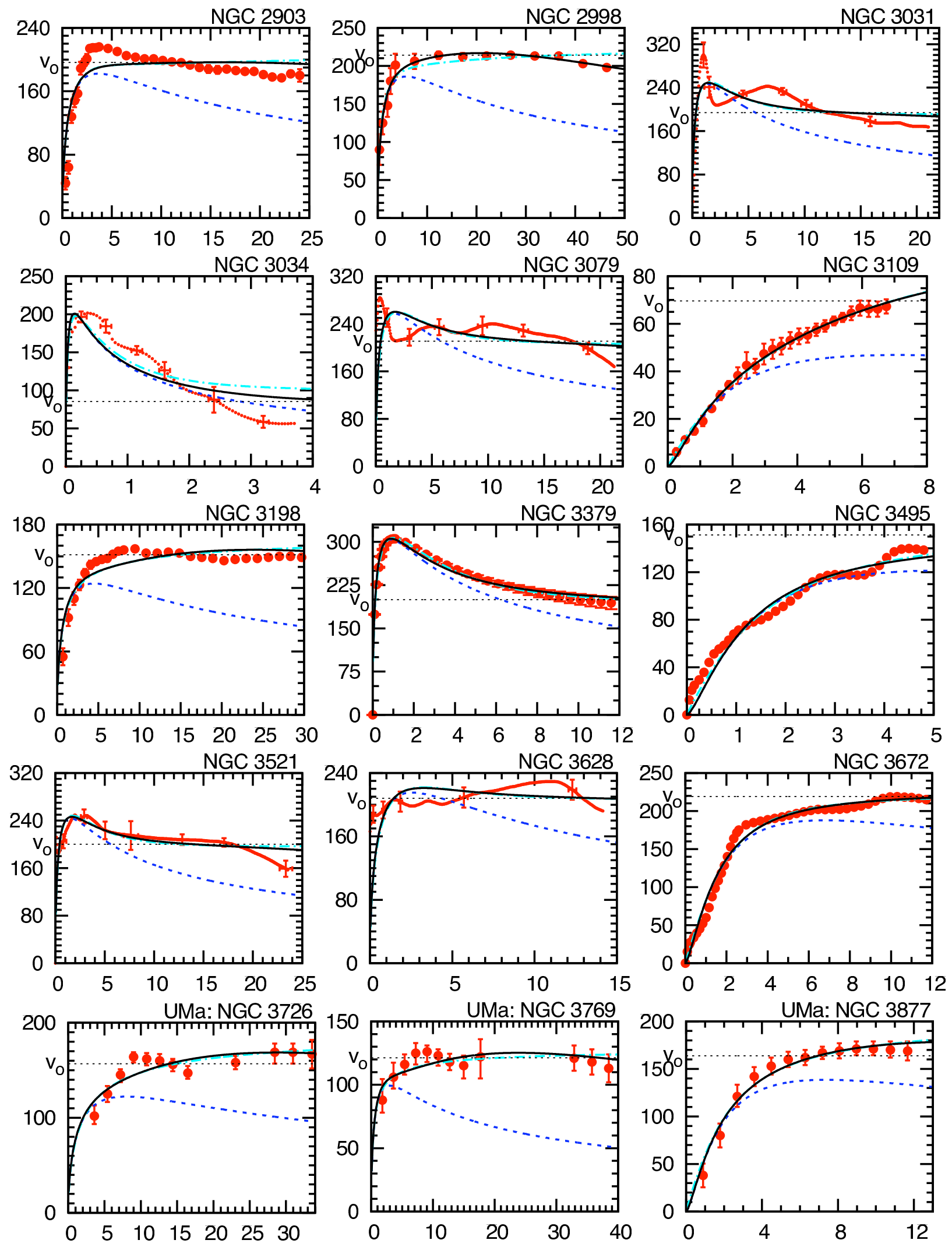

Fig. 2 Continued.- Parametric Galaxy Rotation Curve Fits 

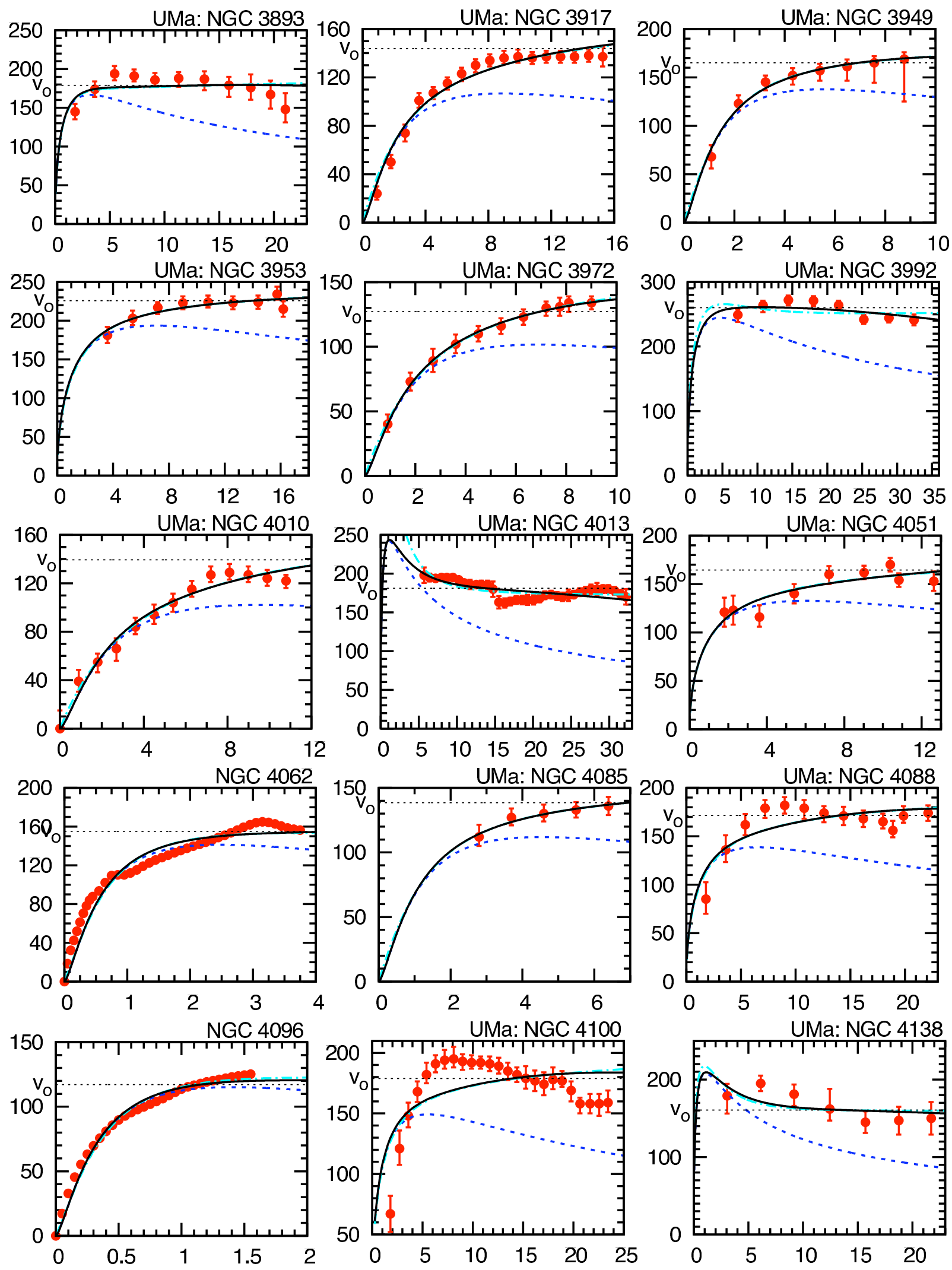

Fig. 2 Continued.- Parametric Galaxy Rotation Curve Fits 

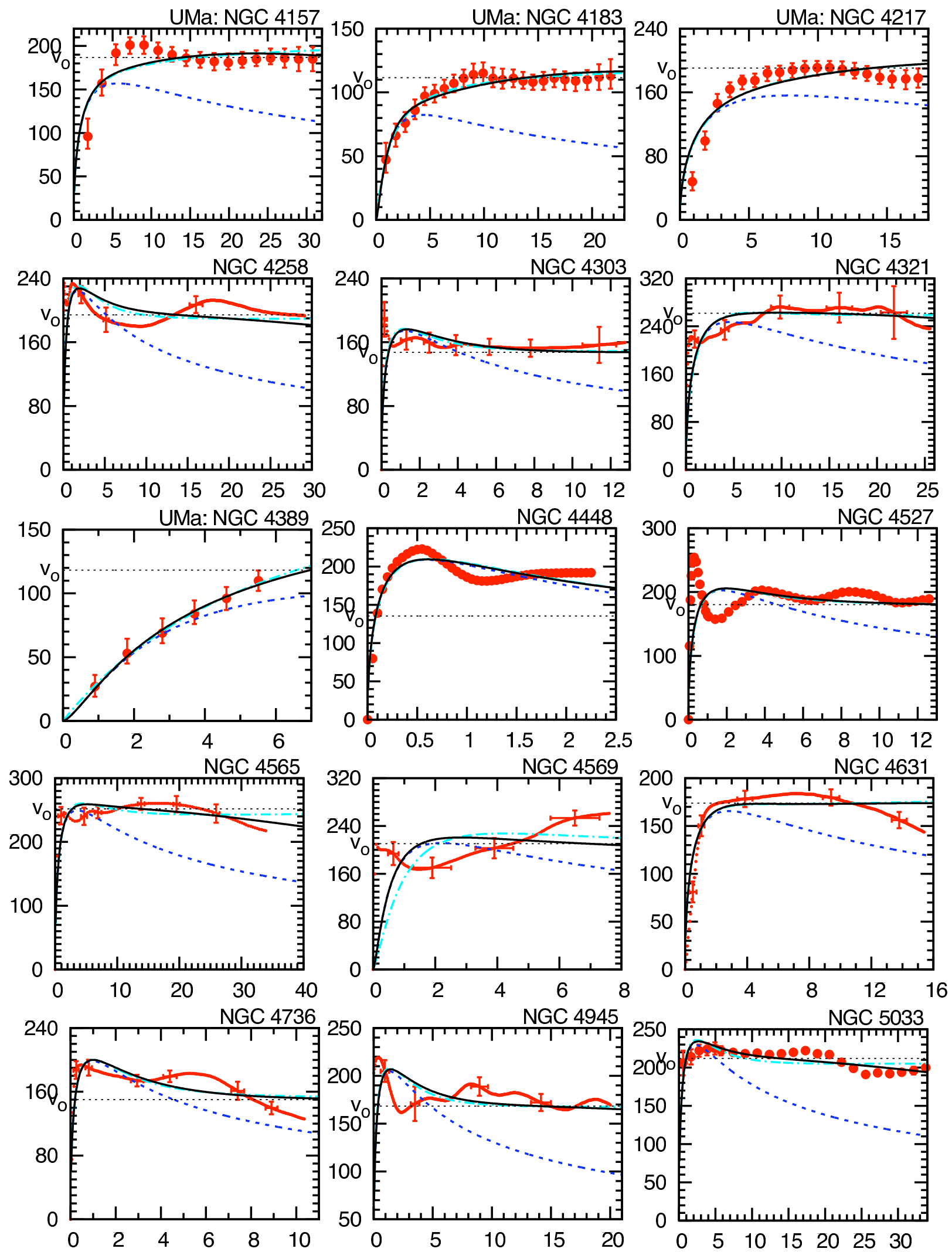

Fig. 2 Continued.- Parametric Galaxy Rotation Curve Fits 

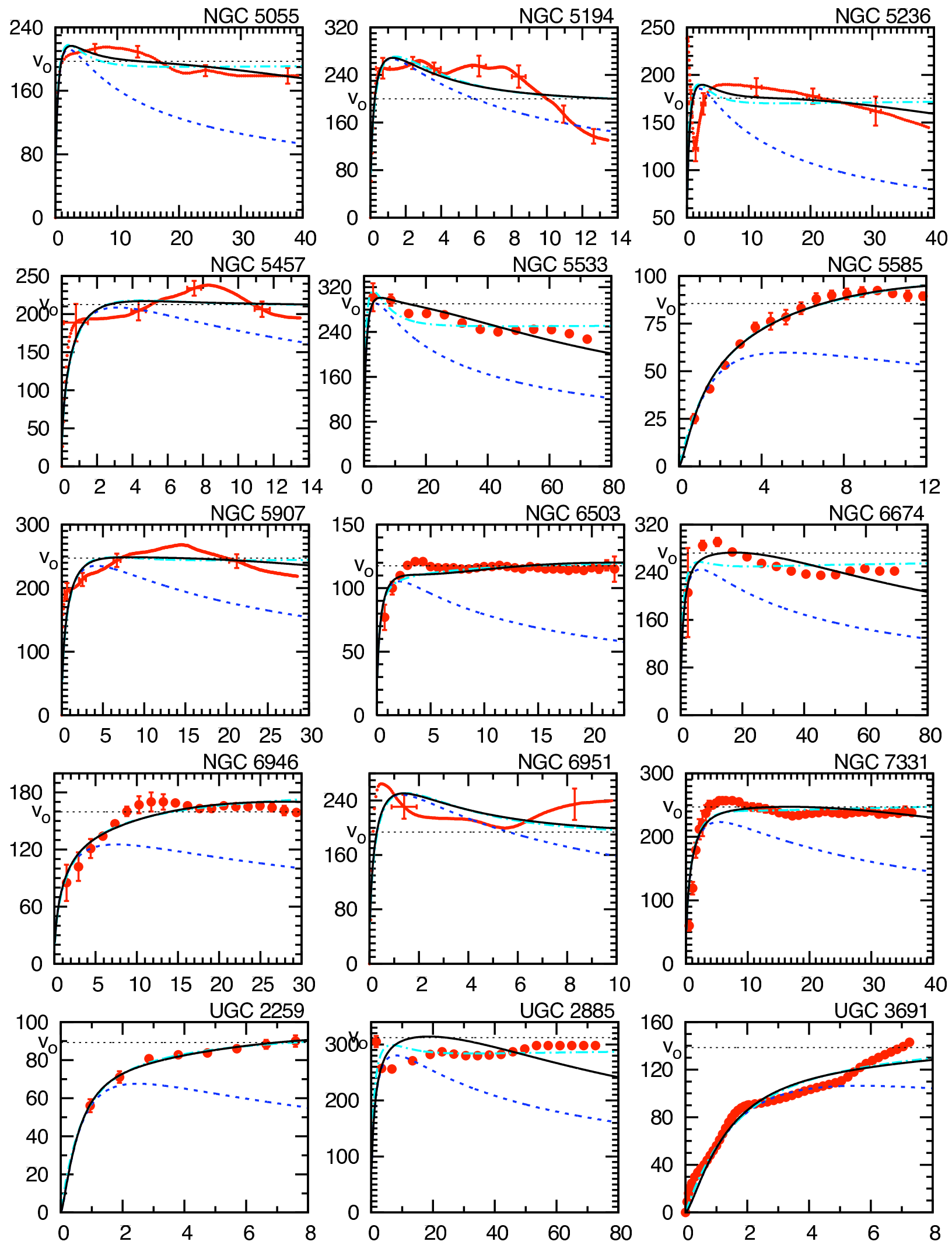

Fig. 2 Continued.- Parametric Galaxy Rotation Curve Fits 

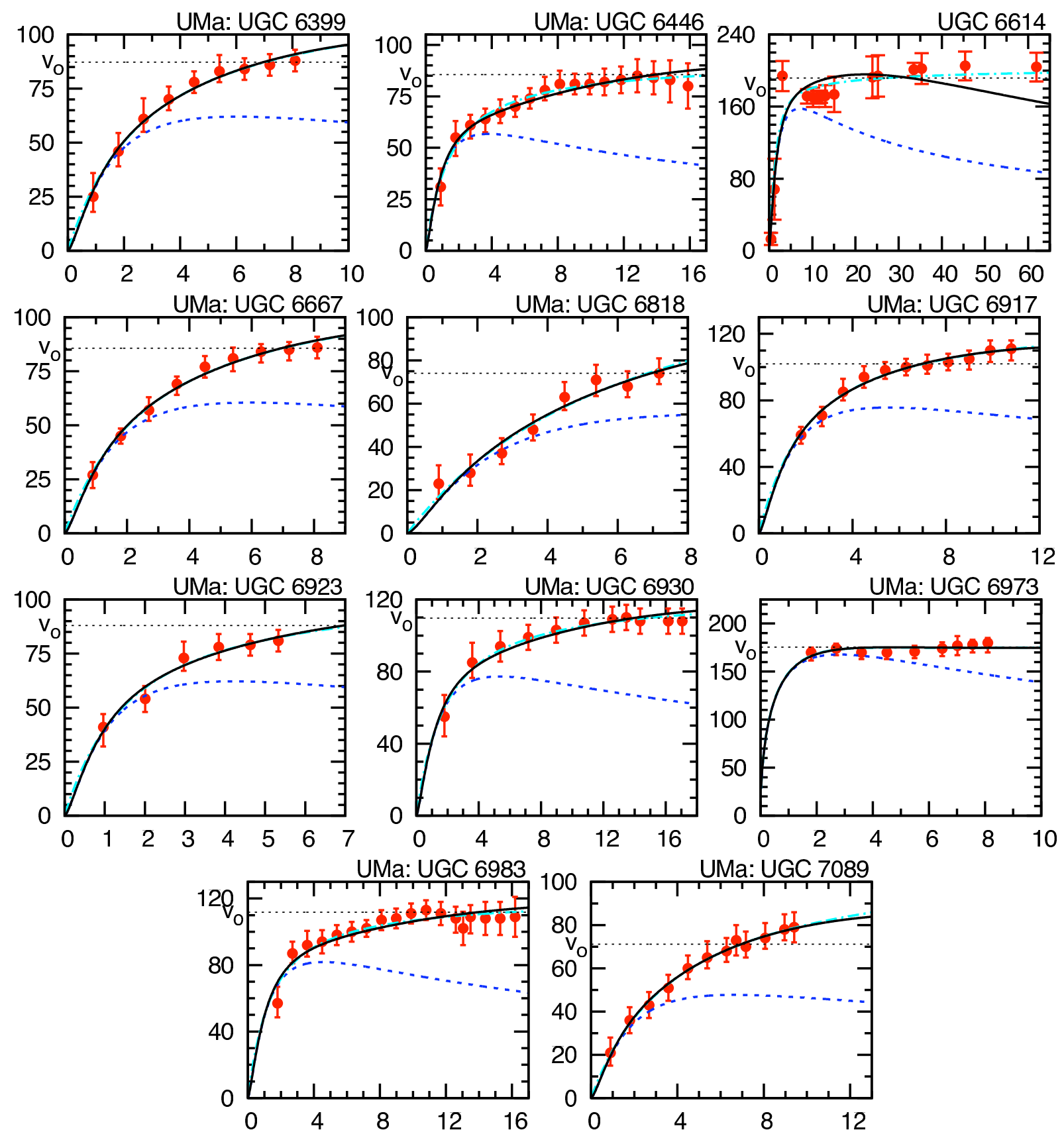

Fig. 2 Continued.- Parametric Galaxy Rotation Curve Fits 

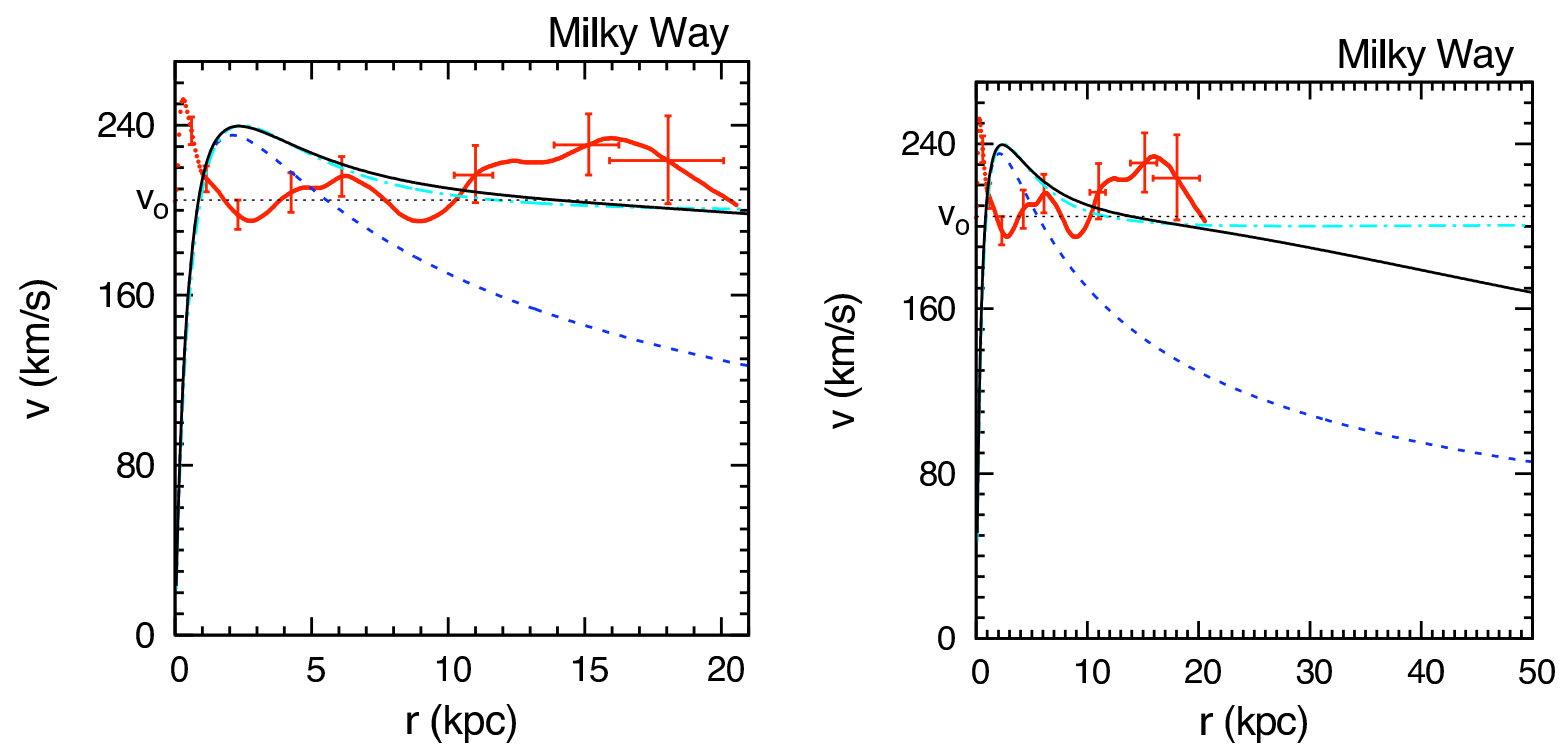

Fig. 3.- High Resolution rotation curve for the Milky Way. Both rotation curves are the same best fit to a parametric mass distribution (independent of luminosity observations) a two parameter fit to the total galactic mass, $M$, and a core radius, $r_{c}$. The red points are the observations - error bars are shown as region specific. The solid black line is the rotation curve determined from MSTG, the dash-dot cyan line is the rotation curve determined from MOND. The horizontal dotted black line is the MSTG predicted value of the measured "flat rotation velocity", $v_{0}$ of equation equation (35). The remaining curve - the short dashed blue line is the Newtonian galaxy rotation curve. The first rotation curve (leftmost) is plotted out to the edge of the visible stars in the galaxy at a distance of $r_{\text {out }} \approx 20.5 \mathrm{kpc}$. The second rotation curve (rightmost) is plotted out to $50 \mathrm{kpc}$ in order to distinguish the MSTG and MOND predictions. The numerical results of the fit are presented in Table 3. 

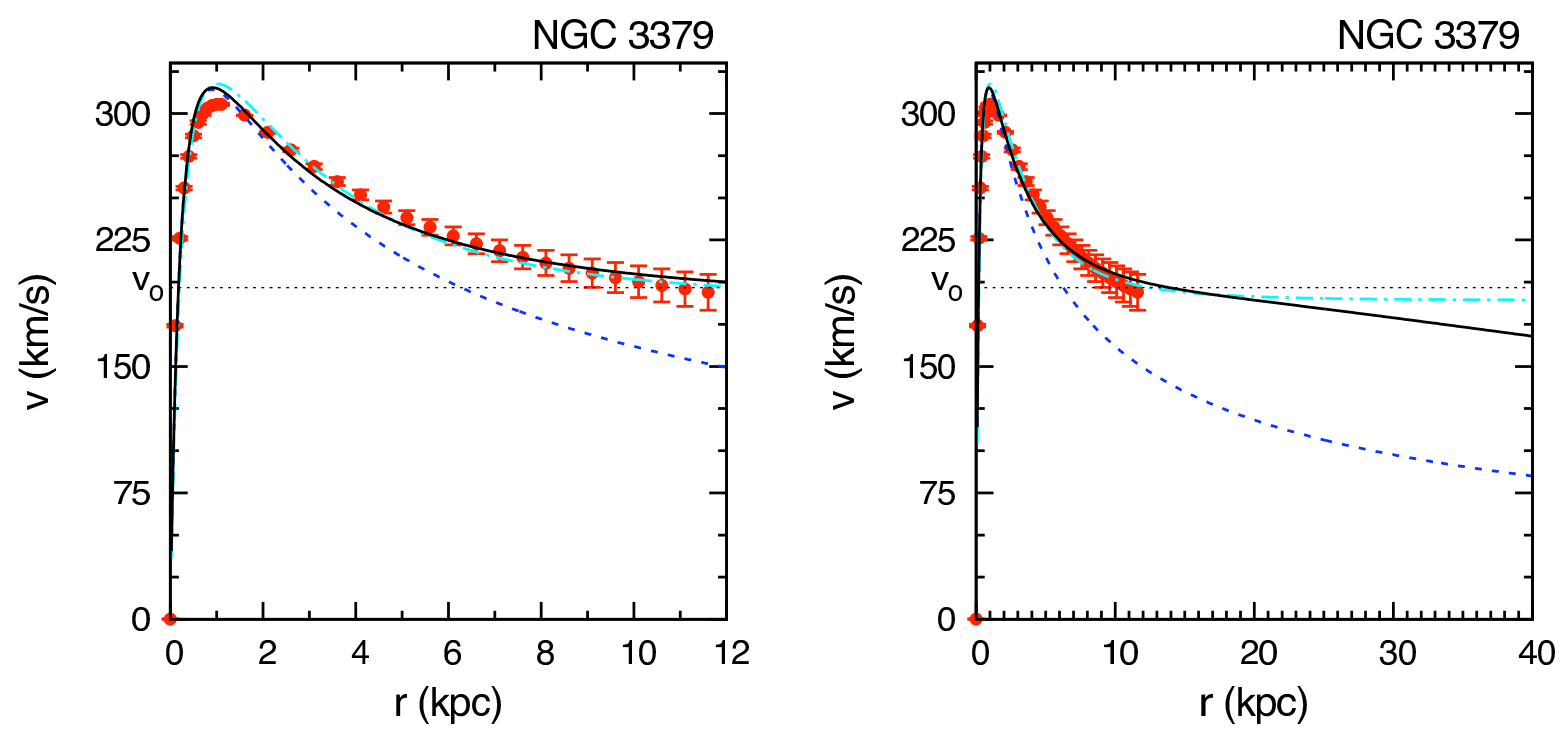

Fig. 4.- Rotation curve for the elliptical galaxy NGC 3379. Both rotation curves are the same best fit to a parametric mass distribution (independent of luminosity observations) a two parameter fit to the total galactic Mass, $M$, and a core radius, $r_{c}$. The red points (with error bars) are samplings of the circular velocity profile constrained by orbit modeling according to Romanowsky et al. (2003, 2004). The solid black line is the rotation curve determined from MSTG, the dash-dot cyan line is the rotation curve determined from MOND. The horizontal dotted black line is the MSTG predicted value of the measured "flat rotation velocity", $v_{0}$ of equation equation (35). The remaining curve - the short dashed blue line is the Newtonian galaxy rotation curve. The first rotation curve (leftmost) is plotted out to the edge of the visible stars in the galaxy at a distance of $r_{\text {out }} \approx 12 \mathrm{kpc}$. The second rotation curve (rightmost) is plotted out to $40 \mathrm{kpc}$ in order to distinguish the MSTG and MOND predictions. The numerical results of the fit are presented in Table 3. 

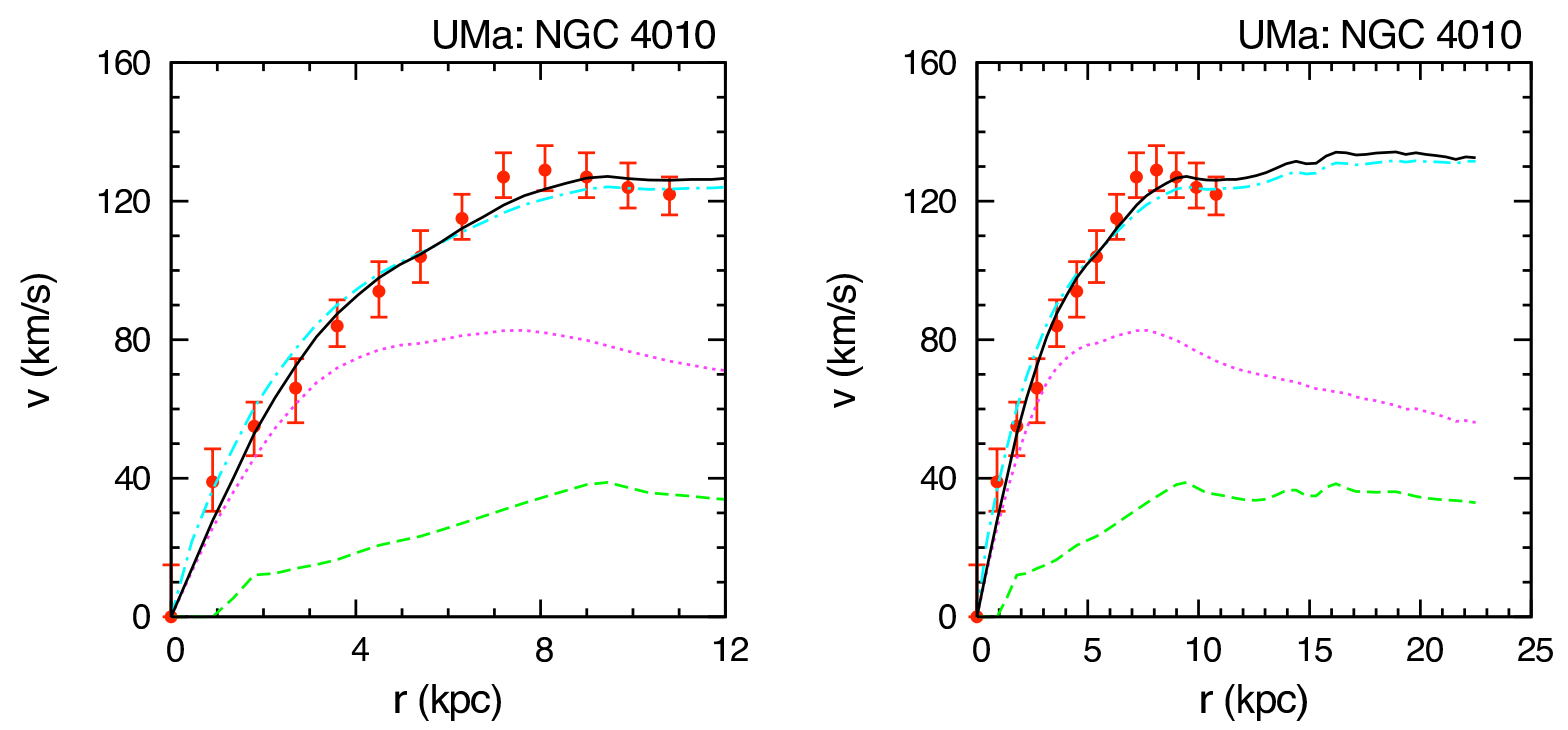

Fig. 5.- Rotation curve for NGC 4010 in the Ursa Major cluster of galaxies reproduced from the original photometry of Verheijen (2001); Verheijen \& Sancisi (2001) - accounting for the revised distance estimate to UMa from 15.5 Mpc to 18.6 Mpc. Both rotation curves are the same best fit via the single parameter $(M / L)_{\text {stars }}$ based on the $K$-band photometric observations of the gaseous (HI plus He) and luminous stellar disks for MSTG and MOND. In every case, the radial coordinate (horizontal axis) is given in kpc and the rotation velocity (vertical axis) in km/s. The red points with error bars are the observations, the solid black line is the rotation curve determined from MSTG, the dash-dot cyan line is the rotation curve determined from MOND. The other curves are the Newtonian rotation curves of the various separate components: the long dashed green line is the rotation curve of the gaseous disk (HI plus He); the dotted magenta curve is that of the luminous stellar disk. The first rotation curve (leftmost) is plotted out to the edge of the visible stars in the galaxy at a distance of $r_{\text {out }} \approx 10.8 \mathrm{kpc}$. The second rotation curve (rightmost) is plotted out to $\approx 23 \mathrm{kpc}$ - the extent of the HI and $K$-band data - in order to extend and distinguish the MSTG and MOND "flat rotation velocity" predictions. The numerical results of the fit are presented in Table 2. 

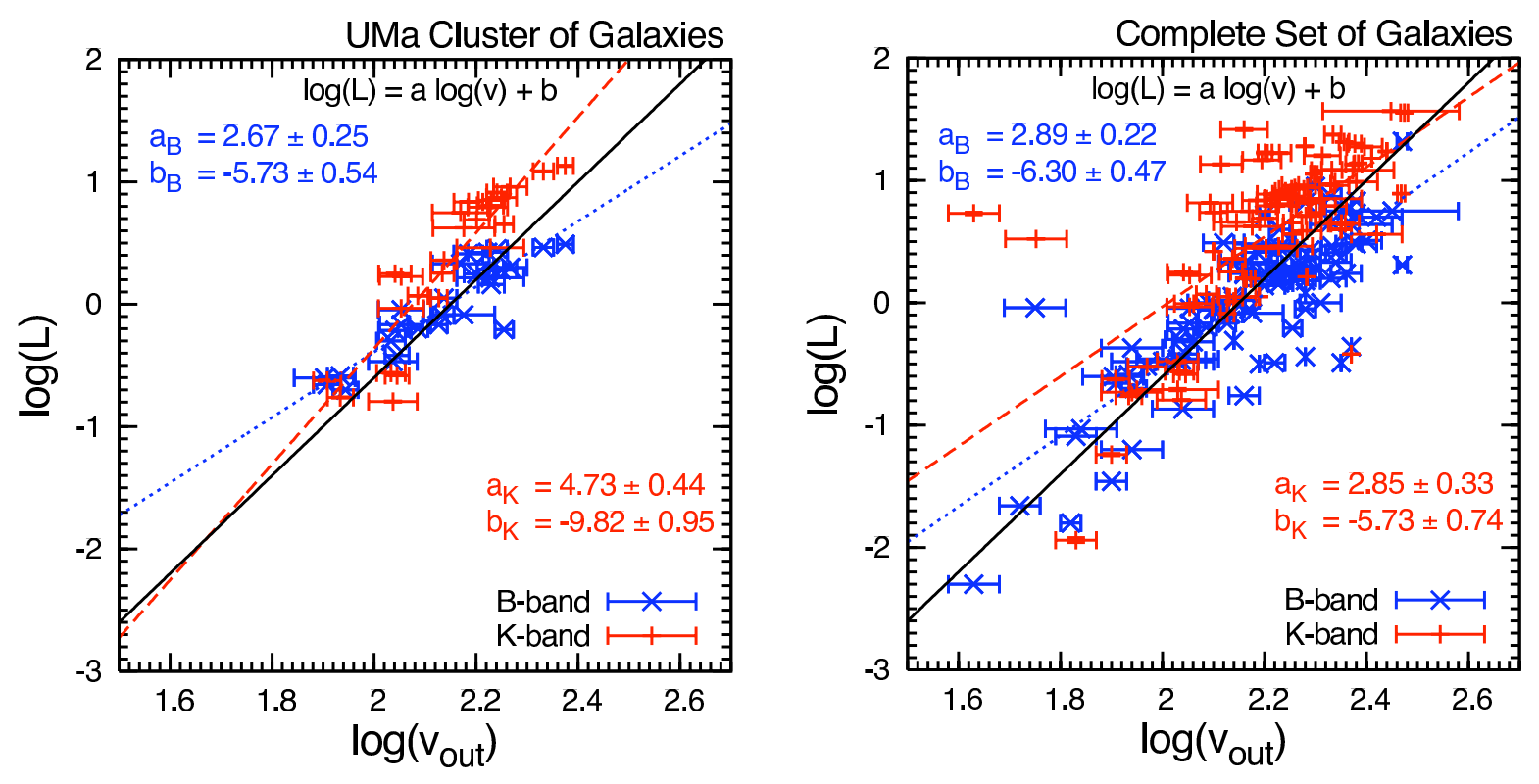

Fig. 6.- Observed $B$ - and $K$-band Tully-Fisher relation for the UMa cluster of galaxies, and the complete sample of galaxies. The vertical axis is the (base 10) logarithm of the observed galaxy luminosity (in $10^{10} L_{\odot}$ ); and the horizontal axis is the (base 10) logarithm of the observed rotational velocity (in $\mathrm{km} / \mathrm{s}$ ) at the maximum observed radius. In both plots, the blue $\times$-points are the observed $B$-band luminosity data and the red + -points are the observed $K$-band luminosity data. In all cases, the Tully-Fisher relation is parametrized by $\log (L) \equiv \operatorname{alog}(v)+b$. In both plots, the blue dotted lines are the best fit $B$-band TullyFisher relation and the red dashed lines are the best fit $K$-band Tully-Fisher relation. The best fit results using a nonlinear least-squares fitting routine including estimated errors are summarized in Table 4. The solid black line is the MOND prediction with $\langle M / L\rangle \equiv 1$. 

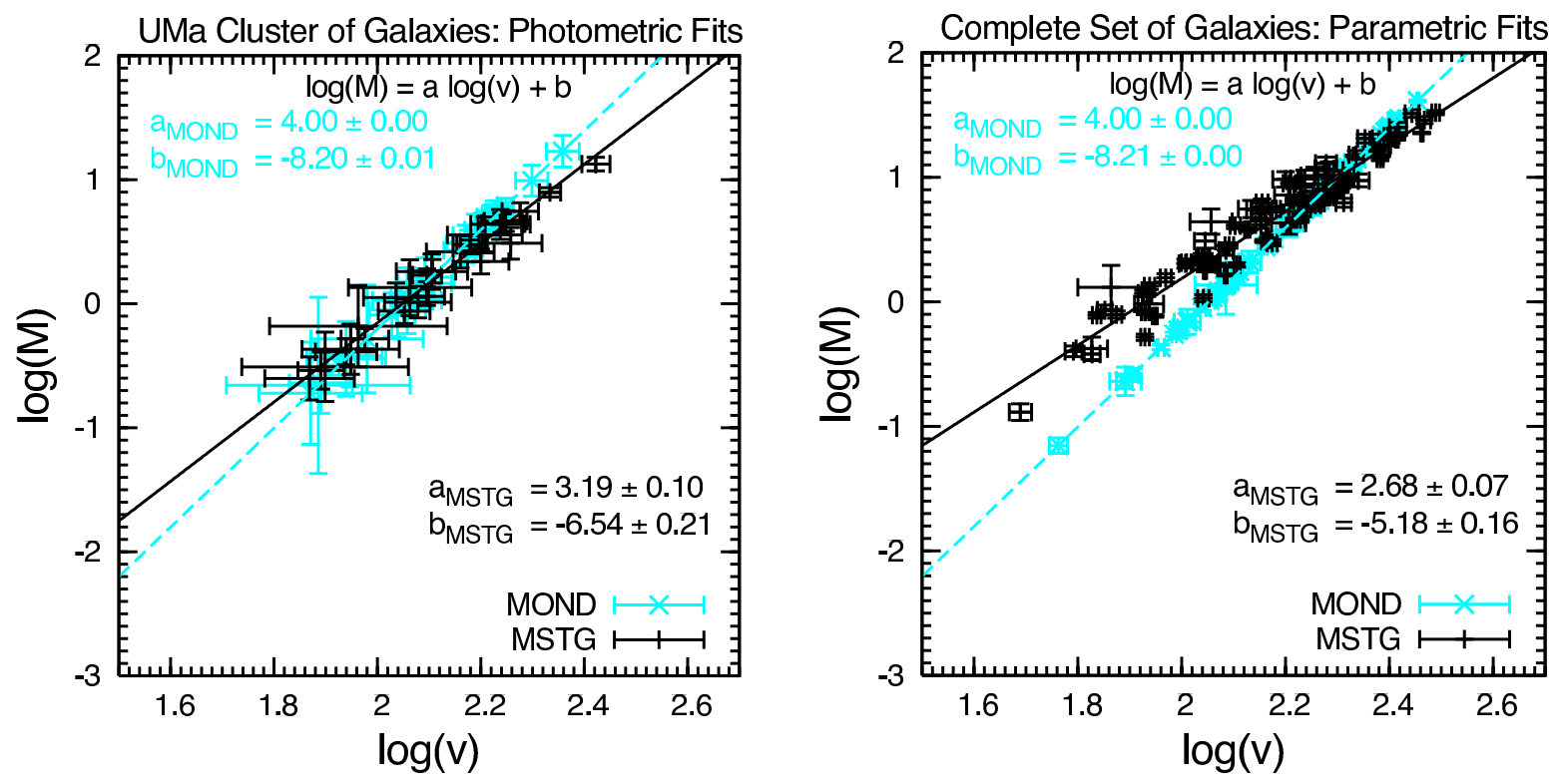

Fig. 7.- Actual Tully-Fisher relation for the UMa cluster of galaxies' photometry, and the complete sample of galaxies resulting from the parametric fits. The first curve (leftmost) is the combined HSB, LSB and Dwarf galaxies fit to the photometric data of the UMa cluster of galaxies corresponding to those galaxies of Fig. 1 labeled by $U M a$ and Table 2. The second curve (rightmost) is the combined HSB, LSB and Dwarf galaxies fit to the parametric data of the complete sample corresponding to Fig. 2 and Table 3. The vertical axes are the (base 10) logarithm of the total mass of the galaxy (in $10^{10} M_{\odot}$ ) resulting from the fits. The horizontal axes are the (base 10) logarithm of the flat rotational velocity (in $\mathrm{km} / \mathrm{s}$ ) as determined from the fits $-v_{0}$ in MSTG and $v_{c}$ in MOND. In all plots, the cyan $\times$-points are the MOND results, and the black +-points are the MSTG results. In all cases, the Tully-Fisher relation is parametrized by $\log (M) \equiv a \log (v)+b$; and the results of the fits are shown for MSTG and MOND. The dashed cyan line is the best fit solution for the MOND results, and the solid black line is the best fit solution for MSTG results. The best fit results using a nonlinear least-squares fitting routine including estimated errors are summarized in Table 4. 
Table 1. Galaxy Properties of the Complete Sample

\begin{tabular}{|c|c|c|c|c|c|c|c|c|c|}
\hline Galaxy & $\begin{array}{c}\text { Type } \\
(2)\end{array}$ & $\begin{array}{c}\text { Surface } \\
\text { Brightness } \\
(3)\end{array}$ & $\begin{array}{c}L_{B} \\
\left(10^{10} L_{\odot}\right) \\
(4)\end{array}$ & $\begin{array}{c}L_{K} \\
\left(10^{10} L_{\odot}\right) \\
(5)\end{array}$ & $\begin{array}{c}r_{\text {out }} \\
(\mathrm{kpc}) \\
(6)\end{array}$ & $\begin{array}{c}v_{\text {out }} \\
\left(\mathrm{km} \mathrm{s}^{-1}\right) \\
(7)\end{array}$ & $\begin{array}{c}\text { Distance } \\
(\mathrm{Mpc}) \\
(8)\end{array}$ & $\begin{array}{c}\text { Redshift } \\
\text { (9) }\end{array}$ & $\begin{array}{l}\text { Ref } \\
(10)\end{array}$ \\
\hline DDO 154 & IB & LSB & 0.005 & 5.392 & 7.6 & $43.1 \pm 2.0$ & 4.0 & 0.000103 & 1 \\
\hline DDO 168 & Irr & LSB & 0.022 & $\ldots$ & 3.7 & $52.0 \pm 2.0$ & 3.8 & 0.000640 & 2 \\
\hline DDO 170 & $\mathrm{Im}$ & $\ldots$ & 0.016 & $\ldots$ & 9.6 & $66.0 \pm 1.0$ & 12.0 & 0.003119 & 1 \\
\hline F563-1 & $\mathrm{Sm}$ & LSB & 0.135 & 0.100 & 17.5 & $110.9 \pm 7.0$ & 45.0 & $\ldots$ & 3 \\
\hline F568-3 & $\mathrm{Sd}$ & LSB & 0.331 & 0.272 & 11.2 & $101.0 \pm 10.2$ & 77.0 & $\ldots$ & 3 \\
\hline F571-8 & $\ldots$ & LSB & 0.174 & $\ldots$ & 14.0 & $143.9 \pm 5.0$ & 48.0 & $\ldots$ & 3 \\
\hline F583-1 & $\mathrm{Sm}$ & LSB & 0.063 & 0.054 & 14.0 & $86.9 \pm 5.6$ & 32.0 & $\ldots$ & 3 \\
\hline F583-4 & $\mathrm{Sc}$ & LSB & 0.093 & 0.072 & 6.7 & $69.9 \pm 4.9$ & 49.0 & $\ldots$ & 3 \\
\hline IC 342 & $\mathrm{Sc}$ & HSB & 6.607 & 5.126 & 19.3 & $191.1 \pm 12.7$ & 3.9 & 0.000103 & 4 \\
\hline Milky Way & $\mathrm{Sb}$ & HSB & $\ldots$ & $\ldots$ & 20.5 & $202.6_{-20.3}^{+21.1}$ & $\ldots$ & $\ldots$ & 4 \\
\hline NGC 55 & $\mathrm{SBm}$ & LSB & 0.430 & 0.187 & 9.8 & $86.5 \pm 5.5$ & 1.6 & 0.000430 & 2 \\
\hline NGC 224 & $\mathrm{Sb}$ & HSB & 1.738 & 4.443 & 31.3 & $227.9 \pm 14.5$ & 0.7 & -0.001001 & 4 \\
\hline NGC 247 & $\mathrm{SBc}$ & LSB & 0.350 & 0.193 & 11.0 & $107.8 \pm 9.0$ & 2.8 & 0.000520 & 2 \\
\hline NGC 253 & $\mathrm{Sc}$ & HSB & 1.585 & 4.482 & 9.7 & $211.5 \pm 12.5$ & 2.5 & 0.000811 & 4 \\
\hline NGC 300 & $\mathrm{Sc}$ & HSB & 0.300 & 0.300 & 12.1 & $93.4 \pm 8.0$ & 2.2 & 0.000480 & 2 \\
\hline NGC 598 & $\mathrm{Sc}$ & LSB & 0.331 & 0.330 & 6.9 & $108.1 \pm 4.0$ & 0.8 & -0.000597 & 4 \\
\hline NGC 660 & $\mathrm{Sc}$ & HSB & 1.047 & 4.557 & 23.3 & $139.6 \pm 14.5$ & 13.0 & 0.002835 & 4 \\
\hline NGC 801 & $\mathrm{Sc}$ & HSB & 7.400 & 23.640 & 59.0 & $216.0 \pm 8.0$ & 80.0 & 0.019227 & 2 \\
\hline NGC 891 & $\mathrm{Sb}$ & HSB & 1.514 & 7.733 & 24.8 & $164.6 \pm 11.5$ & 8.9 & 0.001761 & 4 \\
\hline NGC 1003 & Scd & LSB & 0.437 & 0.382 & 0.8 & $233.6^{\mathrm{a}}$ & 9.5 & 0.002088 & 4 \\
\hline NGC 1068 & $\mathrm{Sb}$ & HSB & 5.623 & 36.796 & 7.3 & $280.8_{-86.5}^{+27.7}$ & 18.1 & 0.003793 & 4 \\
\hline NGC 1097 & $\mathrm{SBb}$ & HSB & 3.236 & 18.762 & 33.2 & $249.1 \pm 20.0$ & 16.0 & 0.004240 & 4 \\
\hline NGC 1365 & $\mathrm{SBb}$ & HSB & 1.000 & 15.974 & 31.1 & $205.7 \pm 16.7$ & 15.6 & 0.005457 & 4 \\
\hline NGC 1417 & $\mathrm{Sb}$ & LSB & $\ldots$ & 15.200 & 9.6 & $255.7^{\mathrm{a}}$ & 54.1 & 0.013569 & 4 \\
\hline NGC 1560 & $\mathrm{Sd}$ & LSB & 0.035 & 0.058 & 8.3 & $78.7 \pm 2.0$ & 3.0 & -0.000120 & 1 \\
\hline NGC 1808 & $\mathrm{SBc}$ & HSB & 1.000 & 6.559 & 16.0 & $123.8 \pm 12.7$ & 11.4 & 0.003319 & 4 \\
\hline NGC 2403 & $\mathrm{Sc}$ & HSB & 0.790 & 0.816 & 19.3 & $134.0 \pm 4.0$ & 3.3 & 0.000437 & 1 \\
\hline NGC 2590 & $\mathrm{Sb}$ & HSB & $\ldots$ & 17.278 & 15.8 & $275.2^{\mathrm{a}}$ & 64.5 & 0.016665 & 4 \\
\hline NGC 2708 & $\mathrm{Sb}$ & HSB & 0.324 & 3.952 & 4.6 & $225.7^{\mathrm{a}}$ & 24.6 & 0.006698 & 4 \\
\hline NGC 2841 & $\mathrm{Sb}$ & HSB & 2.050 & 7.797 & 42.7 & $294.0 \pm 5.0$ & 9.5 & 0.002128 & 1 \\
\hline NGC 2903 & $\mathrm{Sc}$ & HSB & 1.530 & 3.654 & 24.0 & $180.0 \pm 8.0$ & 6.4 & 0.001855 & 1 \\
\hline NGC 2998 & $\mathrm{SBc}$ & HSB & 9.000 & 11.288 & 46.4 & $198.0 \pm 3.0$ & 67.0 & 0.015958 & 2 \\
\hline NGC 3031 & $\mathrm{Sb}$ & HSB & 0.324 & 7.167 & 21.1 & $167.4 \pm 9.0$ & 3.3 & -0.000113 & 4 \\
\hline NGC 3034 & $\mathrm{I}$ & HSB & 0.912 & 3.327 & 3.7 & $56.5 \pm 7.8$ & 3.3 & 0.000677 & 4 \\
\hline NGC 3079 & $\mathrm{Sc}$ & HSB & 3.981 & 7.032 & 21.3 & $167.9 \pm 9.6$ & 15.6 & 0.003723 & 4 \\
\hline NGC 3109 & $\mathrm{SBm}$ & LSB & 0.081 & 0.011 & 6.7 & $67.3 \pm 3.0$ & 1.6 & 0.001344 & 1 \\
\hline NGC 3198 & $\mathrm{Sc}$ & HSB & 0.900 & 1.570 & 29.7 & $149.0 \pm 3.0$ & 9.4 & 0.002212 & 1 \\
\hline NGC 3379 & $\mathrm{E}$ & HSB & 0.891 & 8.712 & 12.0 & $192.5 \pm 11.0$ & 11.0 & 0.003039 & 6 \\
\hline NGC 3495 & $\mathrm{Sd}$ & LSB & 0.490 & 1.018 & 4.8 & $138.5^{\mathrm{a}}$ & 12.8 & 0.003789 & 4 \\
\hline NGC 3521 & $\mathrm{SBb}$ & HSB & 1.380 & 14.648 & 23.6 & $156.8 \pm 13.6$ & 11.4 & 0.002672 & 4 \\
\hline NGC 3628 & $\mathrm{Sb} / \mathrm{I}$ & HSB & 1.514 & 3.871 & 14.2 & $192.0 \pm 12.7$ & 6.7 & 0.002812 & 4 \\
\hline NGC 3672 & $\mathrm{Sc}$ & LSB & 4.571 & 9.267 & 11.8 & $214.7^{\mathrm{a}}$ & 28.4 & 0.006211 & 4 \\
\hline NGC 3726 & $\mathrm{SBc}$ & HSB & 2.650 & 6.216 & 33.6 & $167.0 \pm 15.0$ & 18.6 & 0.002887 & 5 \\
\hline NGC 3769 & $\mathrm{SBb}$ & HSB & 0.680 & 1.678 & 38.5 & $113.0 \pm 11.0$ & 18.6 & 0.002459 & 5 \\
\hline
\end{tabular}


Table 1-Continued

\begin{tabular}{|c|c|c|c|c|c|c|c|c|c|}
\hline $\begin{array}{c}\text { Galaxy } \\
\text { (1) }\end{array}$ & $\begin{array}{c}\text { Type } \\
(2)\end{array}$ & $\begin{array}{c}\text { Surface } \\
\text { Brightness } \\
(3)\end{array}$ & $\begin{array}{c}L_{B} \\
\left(10^{10} L_{\odot}\right) \\
(4)\end{array}$ & $\begin{array}{c}L_{K} \\
\left(10^{10} L_{\odot}\right) \\
(5)\end{array}$ & $\begin{array}{c}r_{\text {out }} \\
(\mathrm{kpc}) \\
(6)\end{array}$ & $\begin{array}{c}v_{\text {out }} \\
\left(\mathrm{km} \mathrm{s}^{-1}\right) \\
(7)\end{array}$ & $\begin{array}{c}\text { Distance } \\
(\mathrm{Mpc}) \\
(8)\end{array}$ & $\begin{array}{c}\text { Redshift } \\
\text { (9) }\end{array}$ & $\begin{array}{l}\text { Ref } \\
(10)\end{array}$ \\
\hline NGC 3877 & $\mathrm{Sc}$ & HSB & 1.940 & 6.396 & 11.7 & $169.0 \pm 10.0$ & 18.6 & 0.002987 & 5 \\
\hline NGC 3893 & $\mathrm{Sc}$ & HSB & 2.140 & 5.598 & 21.1 & $148.0_{-17}^{+21}$ & 18.6 & 0.003226 & 5 \\
\hline NGC 3917 & Scd & LSB & 1.120 & 2.289 & 15.3 & $137.0 \pm 8.0$ & 18.6 & 0.003218 & 5 \\
\hline NGC 3949 & $\mathrm{Sbc}$ & HSB & 1.650 & 2.901 & 8.8 & $169.0_{-44}^{+7}$ & 18.6 & 0.002669 & 5 \\
\hline NGC 3953 & $\mathrm{SBbc}$ & HSB & 2.910 & 12.183 & 16.2 & $215.0 \pm 10.0$ & 18.6 & 0.003510 & 5 \\
\hline NGC 3972 & $\mathrm{Sbc}$ & HSB & 0.680 & 1.124 & 9.0 & $134.0 \pm 5.0$ & 18.6 & 0.002843 & 5 \\
\hline NGC 3992 & $\mathrm{SBbc}$ & HSB & 3.100 & 13.482 & 36.0 & $237.0_{-10}^{+7}$ & 18.6 & 0.003496 & 5 \\
\hline NGC 4010 & $\mathrm{SBd}$ & LSB & 0.630 & 1.169 & 10.8 & $122.0_{-6}^{+5}$ & 18.6 & 0.003008 & 5 \\
\hline NGC 4013 & $\mathrm{Sb}$ & HSB & 1.450 & 7.090 & 32.2 & $170.0 \pm 10.0$ & 18.6 & 0.002773 & 5 \\
\hline NGC 4051 & $\mathrm{SBbc}$ & HSB & 2.580 & 6.856 & 12.6 & $153.0 \pm 10.0$ & 18.6 & 0.002336 & 5 \\
\hline NGC 4062 & $\mathrm{Sc}$ & HSB & 0.316 & 1.131 & 3.8 & $156.3^{\mathrm{a}}$ & 9.7 & 0.002565 & 4 \\
\hline NGC 4085 & $\mathrm{Sc}$ & HSB & 0.810 & 1.797 & 6.4 & $136.0 \pm 7.0$ & 18.6 & 0.002487 & 5 \\
\hline NGC 4088 & $\mathrm{SBc}$ & HSB & 2.830 & 8.176 & 22.1 & $174.0 \pm 8.0$ & 18.6 & 0.002524 & 5 \\
\hline NGC 4096 & $\mathrm{Sc}$ & HSB & 0.891 & 2.610 & 1.6 & $125.3^{\mathrm{a}}$ & 12.2 & 0.001888 & 4 \\
\hline NGC 4100 & $\mathrm{Sbc}$ & HSB & 1.770 & 4.909 & 23.5 & $159.0_{-8}^{+10}$ & 18.6 & 0.003584 & 5 \\
\hline NGC 4138 & $\mathrm{Sa}$ & HSB & 0.820 & 4.203 & 21.7 & $150.0 \pm 21.0$ & 18.6 & 0.002962 & 5 \\
\hline NGC 4157 & $\mathrm{Sb}$ & HSB & 2.000 & 9.098 & 30.8 & $185.0 \pm 14.0$ & 18.6 & 0.002583 & 5 \\
\hline NGC 4183 & Scd & LSB & 0.900 & 0.924 & 21.7 & $113.0_{-10}^{+13}$ & 18.6 & 0.003102 & 5 \\
\hline NGC 4217 & $\mathrm{Sb}$ & HSB & 1.900 & 7.442 & 17.3 & $178.0 \pm 12.0$ & 18.6 & 0.003426 & 5 \\
\hline NGC 4258 & $\mathrm{SBc}$ & HSB & 2.692 & 6.579 & 29.2 & $193.1 \pm 10.6$ & 6.6 & 0.001494 & 4 \\
\hline NGC 4303 & $\mathrm{Sc}$ & HSB & 3.020 & 2.793 & 12.8 & $159.6 \pm 22.5$ & 8.1 & 0.005224 & 4 \\
\hline NGC 4321 & $\mathrm{Sc}$ & HSB & 4.365 & 12.102 & 25.6 & $236.1 \pm 44.0$ & 15.0 & 0.005240 & 4 \\
\hline NGC 4389 & $\mathrm{SBbc}$ & HSB & 0.610 & 1.782 & 5.5 & $110.0 \pm 8.0$ & 18.6 & 0.002396 & 5 \\
\hline NGC 4448 & SBab & HSB & 0.363 & 1.634 & 2.3 & $192.0^{\mathrm{a}}$ & 9.7 & 0.002205 & 4 \\
\hline NGC 4527 & $\mathrm{Sb}$ & HSB & 1.202 & 18.974 & 12.8 & $190.0^{\mathrm{a}}$ & 22.0 & 0.005791 & 4 \\
\hline NGC 4565 & $\mathrm{Sb}$ & HSB & 2.138 & 9.099 & 34.1 & $217.2 \pm 14.4$ & 10.2 & 0.004103 & 4 \\
\hline NGC 4569 & Sab & HSB & 5.012 & 3.621 & 7.6 & $260.9 \pm 12.5$ & 8.2 & -0.000784 & 4 \\
\hline NGC 4631 & $\mathrm{Sc} / \mathrm{I}$ & HSB & 1.738 & 1.111 & 15.4 & $143.5 \pm 8.5$ & 4.3 & 0.002021 & 4 \\
\hline NGC 4736 & $\mathrm{Sab}$ & HSB & 0.871 & 5.460 & 10.4 & $125.8 \pm 7.9$ & 5.1 & 0.001027 & 4 \\
\hline NGC 4945 & $\mathrm{Sc} / \mathrm{I}$ & HSB & 2.818 & 16.740 & 20.0 & $169.6 \pm 9.0$ & 6.7 & 0.001878 & 4 \\
\hline NGC 5033 & $\mathrm{Sc}$ & HSB & 1.900 & 5.420 & 33.8 & $200.0 \pm 5.0$ & 11.9 & 0.002919 & 2 \\
\hline NGC 5055 & $\mathrm{SBc}$ & HSB & 1.778 & 8.467 & 39.4 & $179.0 \pm 10.0$ & 8.0 & 0.001681 & 4 \\
\hline NGC 5194 & $\mathrm{Sc}$ & HSB & 3.090 & 13.515 & 13.5 & $130.4 \pm 12.3$ & 9.6 & 0.001544 & 4 \\
\hline NGC 5236 & $\mathrm{SBc}$ & HSB & 2.089 & 26.057 & 39.3 & $144.4 \pm 15.2$ & 8.9 & 0.001711 & 4 \\
\hline NGC 5457 & $\mathrm{Sc}$ & HSB & 2.344 & 7.012 & 13.5 & $195.0 \pm 10.2$ & 7.2 & 0.000804 & 4 \\
\hline NGC 5533 & Sab & HSB & 5.600 & 20.604 & 72.2 & $227.0 \pm 5.0$ & 54.0 & 0.012896 & 2 \\
\hline NGC 5585 & SBcd & HSB & 0.240 & 0.212 & 11.9 & $89.4 \pm 2.0$ & 7.6 & 0.001017 & 2 \\
\hline NGC 5907 & $\mathrm{Sc}$ & HSB & 2.570 & 6.181 & 28.6 & $218.5 \pm 10.8$ & 11.6 & 0.002225 & 4 \\
\hline NGC 6503 & $\mathrm{Sc}$ & HSB & 0.480 & 0.985 & 22.1 & $115.0 \pm 10.0$ & 5.9 & 0.000200 & 1 \\
\hline NGC 6674 & $\mathrm{SBb}$ & HSB & 6.800 & 13.757 & 69.2 & $242.0 \pm 4.0$ & 49.0 & 0.011438 & 2 \\
\hline NGC 6946 & SABcd & HSB & 5.300 & 16.792 & 29.4 & $159.0 \pm 5.0$ & 10.1 & 0.000160 & 2 \\
\hline NGC 6951 & $\mathrm{Sbc}$ & HSB & 3.020 & 9.741 & 9.8 & $239.7 \pm 23.1$ & 18.0 & 0.004750 & 4 \\
\hline NGC 7331 & $\mathrm{Sb}$ & HSB & 5.400 & 19.969 & 36.0 & $238.0 \pm 7.0$ & 14.9 & 0.002722 & 1 \\
\hline UGC 2259 & SBcd & HSB & 0.100 & 0.008 & 7.6 & $90.0 \pm 3.0$ & 9.8 & 0.001945 & 1 \\
\hline
\end{tabular}


Table 1-Continued

\begin{tabular}{|c|c|c|c|c|c|c|c|c|c|}
\hline $\begin{array}{c}\text { Galaxy } \\
\text { (1) }\end{array}$ & $\begin{array}{c}\text { Type } \\
(2)\end{array}$ & $\begin{array}{c}\text { Surface } \\
\text { Brightness } \\
(3)\end{array}$ & $\begin{array}{c}L_{B} \\
\left(10^{10} L_{\odot}\right) \\
(4)\end{array}$ & $\begin{array}{c}L_{K} \\
\left(10^{10} L_{\odot}\right) \\
(5)\end{array}$ & $\begin{array}{c}r_{\text {out }} \\
(\mathrm{kpc}) \\
(6)\end{array}$ & $\begin{array}{c}v_{\text {out }} \\
\left(\mathrm{km} \mathrm{s}^{-1}\right) \\
(7)\end{array}$ & $\begin{array}{c}\text { Distance } \\
(\mathrm{Mpc}) \\
(8)\end{array}$ & $\begin{array}{c}\text { Redshift } \\
\text { (9) }\end{array}$ & $\begin{array}{l}\text { Ref } \\
\text { (10) }\end{array}$ \\
\hline UGC 2885 & $\mathrm{SBc}$ & HSB & 21.000 & 35.808 & 72.0 & $298.0 \pm 5.0$ & 79.0 & 0.019353 & 2 \\
\hline UGC 3691 & Scd & HSB & 1.698 & 1.573 & 7.2 & $144.1^{\mathrm{a}}$ & 30.0 & 0.007348 & 4 \\
\hline UGC 6399 & $\mathrm{Sm}$ & HSB & 0.200 & $\ldots$ & 8.1 & $88.0 \pm 5.0$ & 18.6 & 0.002640 & 5 \\
\hline UGC 6446 & $\mathrm{Sd}$ & LSB & 0.250 & $\ldots$ & 15.9 & $80.0 \pm 11.0$ & 18.6 & 0.002149 & 5 \\
\hline UGC 6614 & $\ldots$ & LSB & $\ldots$ & 7.157 & 61.9 & $203.9 \pm 16.0$ & 85.0 & 0.021185 & 3 \\
\hline UGC 6667 & Scd & LSB & 0.260 & 0.173 & 8.1 & $86.0 \pm 5.0$ & 18.6 & 0.003246 & 5 \\
\hline UGC 6818 & $\mathrm{Sd}$ & LSB & 0.180 & $\ldots$ & 7.2 & $74.0_{-5}^{+7}$ & 18.6 & 0.002696 & 5 \\
\hline UGC 6917 & $\mathrm{SBd}$ & LSB & 0.380 & 0.260 & 10.8 & $111.0_{-7}^{+5}$ & 18.6 & 0.003038 & 5 \\
\hline UGC 6923 & $\mathrm{Sdm}$ & LSB & 0.220 & 0.237 & 5.3 & $81.0 \pm 5.0$ & 18.6 & 0.003556 & 5 \\
\hline UGC 6930 & $\mathrm{Sd}$ & LSB & 0.500 & 0.275 & 17.1 & $108.0 \pm 7.0$ & 18.6 & 0.002592 & 5 \\
\hline UGC 6973 & $\mathrm{Sab}$ & HSB & 0.620 & 4.513 & 8.1 & $180.0_{-10}^{+5}$ & 18.6 & 0.002337 & 5 \\
\hline UGC 6983 & SBcd & LSB & 0.340 & 0.160 & 16.2 & $109.0 \pm 12.0$ & 18.6 & 0.003609 & 5 \\
\hline UGC 7089 & $\mathrm{Sdm}$ & LSB & 0.440 & $\ldots$ & 9.4 & $79.0 \pm 7.0$ & 18.6 & 0.002568 & 5 \\
\hline
\end{tabular}

Note. - Relevant galaxy properties of the complete sample: Column (1) is the NGC/UGC galaxy number. Column (2) is the galaxy morphological type. Column (3) is the surface brightness - denoted LSB if the galaxy central surface brightness is low $\left(\mu_{0} \gtrsim 23 \mathrm{mag} / \operatorname{arcsec}^{2}\right)$ or HSB if the galaxy central surface brightness is otherwise high. Column (4) is B-band luminosity data taken from the original references except for Sofue (1996) and Romanowsky et al. (2003) which are taken from Tully (1988); and Column (5) is the $K$-band luminosity data converted from the 2MASS $K$-band apparent magnitude via equation (38) except for the Schombert F-type galaxies, which are taken from the original reference. Column (6) is the outermost observed radial position in the rotation velocity data; and Column (7) is the observed velocity at the outermost observed radial position. Column (8) is the distance to the galaxy adopted from the original references; and Column (9) is the observed redshift taken from the NASA/IPAC Extragalactic Database. Column (10) provides the primary original references.

${ }^{a}$ Error bars were not part of the available high resolution rotation curve data for this galaxy.

References. - (1) Begeman, Broeils \& Sanders 1991; (2) Sanders 1996; (3) de Blok \& McGaugh 1998; (4) Verheijen \& Sancisi 2001; (5) Sofue 1996; (6) Romanowsky 2003. 
Table 2. UMa Photometric Rotation Curve Fit Results

\begin{tabular}{|c|c|c|c|c|c|c|c|c|}
\hline Galaxy & $\begin{array}{c}M_{\text {gas }} \\
\left(10^{10} M_{\odot}\right) \\
(2)\end{array}$ & $\begin{array}{c}z_{0} \\
(\mathrm{kpc}) \\
(3)\end{array}$ & $\begin{array}{c}M_{\text {disk }} \\
\left(10^{10} M_{\odot}\right) \\
(4)\end{array}$ & $\begin{array}{c}\text { MSTG } \\
M \\
\left(10^{10} M_{\odot}\right) \\
(5)\end{array}$ & $\begin{array}{c}\underset{v_{0}}{\longrightarrow} \\
\left(\mathrm{km} \mathrm{s}^{-1}\right) \\
(6)\end{array}$ & $\begin{array}{c}\overleftarrow{M_{d i s k}} \\
\left(10^{10} M_{\odot}\right) \\
(7)\end{array}$ & $\begin{array}{c}\text { MOND } \\
M \\
\left(10^{10} M_{\odot}\right) \\
(8)\end{array}$ & $\begin{array}{c}\underset{v_{c}}{\left.\longrightarrow \mathrm{km} \mathrm{s}^{-1}\right)} \\
(9)\end{array}$ \\
\hline \multicolumn{9}{|c|}{ Dwarf (LSB \& HSB) Galaxies } \\
\hline NGC 3877 & 0.21 & 0.56 & $2.86 \pm 0.80$ & $3.07 \pm 0.90$ & $180.9 \pm 25.4$ & $3.71 \pm 1.00$ & $3.92 \pm 1.10$ & $158.3 \pm 11.0$ \\
\hline NGC 3949 & 0.49 & 0.35 & $1.70 \pm 0.42$ & $2.19 \pm 0.50$ & $158.6 \pm 19.7$ & $2.22 \pm 0.80$ & $2.70 \pm 0.75$ & $144.2 \pm 10.0$ \\
\hline NGC 3972 & 0.18 & 0.39 & $0.97 \pm 0.15$ & $1.15 \pm 0.20$ & $124.6 \pm 9.9$ & $1.07 \pm 0.40$ & $1.25 \pm 0.40$ & $118.9 \pm 9.4$ \\
\hline NGC 4085 & 0.16 & 0.31 & $0.87 \pm 0.26$ & $1.03 \pm 0.30$ & $119.7 \pm 17.8$ & $0.94 \pm 0.30$ & $1.10 \pm 0.35$ & $115.1 \pm 9.2$ \\
\hline NGC 4389 & 0.08 & 0.29 & $0.35 \pm 0.15$ & $0.43 \pm 0.20$ & $88.8 \pm 19.2$ & $0.28 \pm 0.30$ & $0.36 \pm 0.25$ & $87.0 \pm 15.3$ \\
\hline UGC 6399 & 0.11 & 0.48 & $0.29 \pm 0.08$ & $0.41 \pm 0.10$ & $87.0 \pm 11.9$ & $0.20 \pm 0.10$ & $0.32 \pm 0.06$ & $84.3 \pm 4.2$ \\
\hline UGC 6667 & 0.12 & 0.58 & $0.40 \pm 0.09$ & $0.52 \pm 0.10$ & $94.5 \pm 10.0$ & $0.26 \pm 0.20$ & $0.38 \pm 0.22$ & $88.5 \pm 12.7$ \\
\hline UGC 6818 & 0.15 & 0.36 & $0.10 \pm 0.04$ & $0.25 \pm 0.10$ & $73.9 \pm 14.7$ & $0.04 \pm 0.20$ & $0.19 \pm 0.18$ & $74.3 \pm 17.1$ \\
\hline UGC 6923 & 0.12 & 0.26 & $0.17 \pm 0.04$ & $0.29 \pm 0.10$ & $77.8 \pm 8.0$ & $0.11 \pm 0.10$ & $0.23 \pm 0.13$ & $77.7 \pm 10.9$ \\
\hline UGC 7089 & 0.19 & 0.62 & $0.12 \pm 0.09$ & $0.31 \pm 0.20$ & $79.2 \pm 29.4$ & $0.03 \pm 0.40$ & $0.22 \pm 0.36$ & $76.9 \pm 31.4$ \\
\hline UGC 6917 & 0.29 & 0.58 & $0.58 \pm 0.13$ & $0.87 \pm 0.20$ & $112.7 \pm 13.0$ & $0.52 \pm 0.30$ & $0.81 \pm 0.33$ & $106.6 \pm 11.0$ \\
\hline \multicolumn{9}{|c|}{ LSB GALAXIES } \\
\hline NGC 3917 & 0.27 & 0.62 & $1.56 \pm 0.32$ & $1.83 \pm 0.40$ & $127.7 \pm 13.3$ & $1.37 \pm 0.70$ & $1.64 \pm 0.66$ & $127.3 \pm 12.7$ \\
\hline UGC 6446 & 0.44 & 0.36 & $0.22 \pm 0.17$ & $0.66 \pm 0.50$ & $91.9 \pm 36.3$ & $0.08 \pm 0.50$ & $0.52 \pm 0.52$ & $95.5 \pm 23.9$ \\
\hline UGC 6983 & 0.42 & 0.53 & $0.70 \pm 0.20$ & $1.12 \pm 0.30$ & $108.7 \pm 15.7$ & $0.47 \pm 0.40$ & $0.89 \pm 0.45$ & $109.3 \pm 13.7$ \\
\hline NGC 4010 & 0.42 & 0.69 & $1.40 \pm 0.44$ & $1.82 \pm 0.60$ & $127.4 \pm 20.2$ & $1.06 \pm 0.40$ & $1.48 \pm 0.38$ & $123.9 \pm 8.0$ \\
\hline NGC 4183 & 0.53 & 0.64 & $0.82 \pm 0.45$ & $1.35 \pm 0.70$ & $115.6 \pm 31.7$ & $0.53 \pm 0.70$ & $1.06 \pm 0.65$ & $114.2 \pm 17.5$ \\
\hline
\end{tabular}

HSB Galaxies 
Table 2-Continued

\begin{tabular}{|c|c|c|c|c|c|c|c|c|}
\hline Galaxy & $\begin{array}{c}M_{\text {gas }} \\
\left(10^{10} M_{\odot}\right) \\
(2)\end{array}$ & $\begin{array}{c}z_{0} \\
(\mathrm{kpc}) \\
(3)\end{array}$ & $\begin{array}{c}M_{\text {disk }} \\
\left(10^{10} M_{\odot}\right) \\
(4)\end{array}$ & $\begin{array}{c}\text { MSTG } \\
M \\
\left(10^{10} M_{\odot}\right) \\
(5)\end{array}$ & $\begin{array}{c}v_{0} \\
\left(\mathrm{~km} \mathrm{~s}^{-1}\right)\end{array}$ & $\begin{array}{c}\overleftarrow{M_{\text {disk }}} \\
\left(10^{10} M_{\odot}\right)\end{array}$ & $\begin{array}{c}\text { MOND } \\
M \\
\left(10^{10} M_{\odot}\right) \\
(8)\end{array}$ & $\begin{array}{c}\longrightarrow \\
v_{c} \\
\left(\mathrm{~km} \mathrm{~s}^{-1}\right) \\
(9)\end{array}$ \\
\hline NGC 3726 & 0.98 & 0.68 & $2.62 \pm 0.87$ & $3.59 \pm 1.20$ & $161.3 \pm 26.9$ & $2.59 \pm 1.40$ & $3.56 \pm 1.40$ & $154.5 \pm 15.2$ \\
\hline NGC 3769 & 0.68 & 0.36 & $1.02 \pm 0.13$ & $1.70 \pm 0.20$ & $124.7 \pm 8.0$ & $0.78 \pm 0.70$ & $1.46 \pm 0.69$ & $123.5 \pm 14.7$ \\
\hline NGC 3893 & 0.76 & 0.49 & $3.67 \pm 0.66$ & $4.43 \pm 0.80$ & $173.9 \pm 15.7$ & $4.24 \pm 1.50$ & $5.00 \pm 1.52$ & $168.2 \pm 12.8$ \\
\hline NGC 3953 & 0.43 & 0.77 & $7.47 \pm 0.71$ & $7.90 \pm 0.70$ & $215.6 \pm 10.2$ & $9.38 \pm 2.80$ & $9.81 \pm 2.81$ & $199.0 \pm 14.3$ \\
\hline NGC 3992 & 0.81 & 0.83 & $12.57 \pm 1.58$ & $13.39 \pm 1.70$ & $264.6 \pm 16.6$ & $16.15 \pm 5.00$ & $16.96 \pm 4.96$ & $228.2 \pm 16.7$ \\
\hline NGC 4013 & 0.45 & 0.41 & $4.19 \pm 0.80$ & $4.64 \pm 0.90$ & $176.9 \pm 16.8$ & $4.58 \pm 0.70$ & $5.03 \pm 0.72$ & $168.4 \pm 6.0$ \\
\hline NGC 4051 & 0.39 & 0.54 & $2.57 \pm 0.29$ & $2.96 \pm 0.30$ & $150.7 \pm 8.4$ & $2.65 \pm 1.10$ & $3.04 \pm 1.06$ & $148.5 \pm 12.9$ \\
\hline NGC 4088 & 1.12 & 0.67 & $3.24 \pm 0.86$ & $4.36 \pm 1.20$ & $172.9 \pm 22.9$ & $3.56 \pm 1.30$ & $4.68 \pm 1.29$ & $165.4 \pm 11.4$ \\
\hline NGC 4100 & 0.45 & 0.51 & $3.96 \pm 0.82$ & $4.41 \pm 0.90$ & $173.7 \pm 18.1$ & $4.43 \pm 1.20$ & $4.88 \pm 1.25$ & $167.1 \pm 10.7$ \\
\hline NGC 4138 & 0.21 & 0.28 & $2.78 \pm 0.42$ & $2.99 \pm 0.50$ & $151.1 \pm 11.5$ & $2.93 \pm 0.70$ & $3.14 \pm 0.70$ & $149.7 \pm 8.4$ \\
\hline NGC 4157 & 1.17 & 0.52 & $4.38 \pm 0.71$ & $5.55 \pm 0.90$ & $188.8 \pm 15.3$ & $4.96 \pm 0.90$ & $6.13 \pm 0.88$ & $176.9 \pm 6.3$ \\
\hline NGC 4217 & 0.37 & 0.58 & $4.12 \pm 0.72$ & $4.49 \pm 0.80$ & $174.8 \pm 15.3$ & $4.66 \pm 1.10$ & $5.03 \pm 1.11$ & $168.4 \pm 9.3$ \\
\hline UGC 6973 & 0.25 & 0.19 & $2.38 \pm 0.72$ & $2.63 \pm 0.80$ & $144.6 \pm 21.7$ & $2.66 \pm 0.20$ & $2.91 \pm 0.16$ & $146.9 \pm 2.0$ \\
\hline
\end{tabular}

Note. - Best fit results of the UMa cluster of galaxies according to both MSTG and MOND via the single parameter $(M / L)_{\text {stars }}$ based on the $K$-band photometric data of the gaseous (HI plus He) and luminous stellar disks, corresponding to the fits (labeled UMa) of Fig. 1 and Fig. 5. Column (1) is the NGC/UGC galaxy number. Column (2) is the mass of the infinitely thin gaseous disk (HI plus He). Column (3) is the $K$-band vertical scale height of the luminous stellar disk. The MSTG best fit results are presented in Columns (4) - (6), where Column (4) is the best fit mass of the luminous stellar disk; Column (5) is the MSTG predicted total mass of the galaxy and is the sum of Column (2) and (4); and Column (6) is the predicted MSTG flat rotation velocity, $v_{0}$, of equation (35). The MOND best results are presented in Columns (7) - (9), where Column (7) is the best fit mass of the luminous stellar disk; Column (8) is the MOND predicted mass of the galaxy and is the sum of Column (2) and (7); and Column (9) is the MOND asymptotic velocity, $v_{c}$ of equation (31). 
Table 3. Parametric Rotation Curve Fit Results

\begin{tabular}{|c|c|c|c|c|c|c|}
\hline Galaxy & $\begin{array}{c}M \\
\left(10^{10} M_{\odot}\right) \\
(2)\end{array}$ & $\begin{array}{c}\text { MSTG } \\
r_{c} \\
(\mathrm{kpc}) \\
(3)\end{array}$ & $\begin{array}{c}\underset{v_{0}}{\longrightarrow} \\
\left(\mathrm{km} \mathrm{s}^{-1}\right) \\
(4)\end{array}$ & $\begin{array}{c}\longleftarrow \\
M \\
\left(10^{10} M_{\odot}\right) \\
(5)\end{array}$ & $\begin{array}{c}\text { MOND } \\
r_{c} \\
(\mathrm{kpc}) \\
(6)\end{array}$ & $\begin{array}{c}\longrightarrow \\
\begin{array}{c}v_{c} \\
\left(\mathrm{~km} \mathrm{~s}^{-1}\right) \\
(7)\end{array}\end{array}$ \\
\hline \multicolumn{7}{|c|}{ Dwarf (LSB \& HSB) Galaxies } \\
\hline DDO $154^{\mathrm{a}}$ & $0.13 \pm 0.02$ & $0.53 \pm 0.07$ & $48.9 \pm 2.4$ & $0.07 \pm 0.01$ & $0.95 \pm 0.12$ & $57.9 \pm 2.3$ \\
\hline DDO 168 & $0.42 \pm 0.09$ & $0.66 \pm 0.08$ & $67.1 \pm 4.7$ & $0.23 \pm 0.06$ & $0.89 \pm 0.14$ & $77.9 \pm 5.5$ \\
\hline DDO 170 & $0.40 \pm 0.04$ & $0.82 \pm 0.07$ & $61.9 \pm 2.3$ & $0.26 \pm 0.03$ & $1.38 \pm 0.10$ & $80.7 \pm 1.9$ \\
\hline F583-4 & $0.38 \pm 0.04$ & $0.57 \pm 0.05$ & $67.2 \pm 2.4$ & $0.23 \pm 0.03$ & $0.77 \pm 0.1$ & $77.5 \pm 2.7$ \\
\hline NGC 55 & $1.17 \pm 0.07$ & $0.99 \pm 0.05$ & $84.4 \pm 2$ & $0.91 \pm 0.07$ & $1.39 \pm 0.08$ & $109.7 \pm 2.1$ \\
\hline NGC 1560 & $0.79 \pm 0.05$ & $0.93 \pm 0.04$ & $74.9 \pm 1.7$ & $0.59 \pm 0.05$ & $1.43 \pm 0.08$ & $98.5 \pm 2.2$ \\
\hline NGC 2708 & $9.43 \pm 1.1$ & $0.66 \pm 0.05$ & $218.7 \pm 10.8$ & $12.97 \pm 1.59$ & $0.79 \pm 0.06$ & $213.4 \pm 6.5$ \\
\hline NGC 3109 & $0.78 \pm 0.04$ & $1.15 \pm 0.04$ & $68.6 \pm 1.3$ & $0.62 \pm 0.04$ & $2 \pm 0.06$ & $99.8 \pm 1.5$ \\
\hline NGC 3877 & $8.65 \pm 0.53$ & $1.31 \pm 0.06$ & $164.8 \pm 4.3$ & $10.34 \pm 0.87$ & $1.52 \pm 0.1$ & $201.7 \pm 4.2$ \\
\hline NGC 3949 & $6.51 \pm 0.3$ & $0.99 \pm 0.03$ & $164.5 \pm 3.2$ & $7.77 \pm 0.41$ & $1.12 \pm 0.04$ & $187.7 \pm 2.5$ \\
\hline NGC 3972 & $4.09 \pm 0.23$ & $1.18 \pm 0.05$ & $126.8 \pm 2.9$ & $4.4 \pm 0.25$ & $1.48 \pm 0.06$ & $162.9 \pm 2.3$ \\
\hline NGC 4062 & $2.98 \pm 0.17$ & $0.43 \pm 0.02$ & $149.4 \pm 3.4$ & $4.07 \pm 0.27$ & $0.53 \pm 0.03$ & $159.7 \pm 2.7$ \\
\hline NGC 4085 & $5.11 \pm 0.54$ & $1.12 \pm 0.07$ & $142 \pm 6.1$ & $5.84 \pm 0.89$ & $1.34 \pm 0.13$ & $174.8 \pm 6.7$ \\
\hline NGC 4096 & $1.07 \pm 0.07$ & $0.24 \pm 0.01$ & $110.1 \pm 2.8$ & $1.21 \pm 0.09$ & $0.26 \pm 0.01$ & $117.9 \pm 2.1$ \\
\hline NGC 4389 & $4.4 \pm 1.02$ & $1.56 \pm 0.18$ & $113.9 \pm 10.6$ & $6.45 \pm 1.7$ & $2.45 \pm 0.28$ & $179.2 \pm 11.8$ \\
\hline NGC 4569 & $6.23 \pm 0.51$ & $0.39 \pm 0.03$ & $205 \pm 7$ & $11.72 \pm 1.18$ & $0.67 \pm 0.06$ & $208.1 \pm 5.2$ \\
\hline NGC 5585 & $1.17 \pm 0.07$ & $0.94 \pm 0.04$ & $85.7 \pm 1.8$ & $0.9 \pm 0.06$ & $1.3 \pm 0.08$ & $109.6 \pm 1.9$ \\
\hline UGC 2259 & $0.77 \pm 0.02$ & $0.48 \pm 0.01$ & $88.8 \pm 1$ & $0.55 \pm 0.02$ & $0.49 \pm 0.02$ & $96.7 \pm 0.9$ \\
\hline UGC 3691 & $2.83 \pm 0.14$ & $0.86 \pm 0.03$ & $123.5 \pm 2.3$ & $2.96 \pm 0.17$ & $1.03 \pm 0.04$ & $147.5 \pm 2.1$ \\
\hline UGC 6399 & $1.34 \pm 0.08$ & $1.05 \pm 0.04$ & $86.7 \pm 2$ & $1.08 \pm 0.09$ & $1.48 \pm 0.08$ & $114.8 \pm 2.5$ \\
\hline UGC 6446 & $0.83 \pm 0.04$ & $0.73 \pm 0.04$ & $85.1 \pm 1.4$ & $0.43 \pm 0.02$ & $0.9 \pm 0.06$ & $91.2 \pm 1.3$ \\
\hline UGC 6818 & $1.31 \pm 0.53$ & $1.5 \pm 0.32$ & $73.1 \pm 10.8$ & $1.37 \pm 0.75$ & $2.78 \pm 0.68$ & $121.7 \pm 16.7$ \\
\hline UGC 6917 & $2.06 \pm 0.11$ & $1.04 \pm 0.05$ & $102.1 \pm 2.2$ & $1.75 \pm 0.11$ & $1.3 \pm 0.07$ & $129.3 \pm 2$ \\
\hline UGC 6923 & $0.96 \pm 0.17$ & $0.74 \pm 0.1$ & $86.5 \pm 5.6$ & $0.69 \pm 0.16$ & $0.91 \pm 0.15$ & $102.5 \pm 5.9$ \\
\hline UGC 7089 & $0.86 \pm 0.08$ & $1.15 \pm 0.07$ & $71.1 \pm 2.3$ & $0.71 \pm 0.08$ & $1.98 \pm 0.14$ & $103.3 \pm 2.9$ \\
\hline
\end{tabular}

\section{LSB GalaXies}

\begin{tabular}{lllllll}
\hline F563-1 & $2.26 \pm 0.16$ & $1.06 \pm 0.07$ & $110.4 \pm 2.7$ & $1.48 \pm 0.15$ & $1.24 \pm 0.14$ & $124.1 \pm 3.1$ \\
F568-3 & $3.08 \pm 0.41$ & $1.58 \pm 0.13$ & $110.9 \pm 5.2$ & $2.09 \pm 0.42$ & $2.13 \pm 0.25$ & $135.2 \pm 6.8$ \\
F571-8 & $5.46 \pm 0.84$ & $1.40 \pm 0.14$ & $141.2 \pm 8.0$ & $4.86 \pm 0.73$ & $1.69 \pm 0.17$ & $167.0 \pm 6.3$
\end{tabular}


Table 3-Continued

\begin{tabular}{|c|c|c|c|c|c|c|}
\hline Galaxy & $\begin{array}{c}M \\
\left(10^{10} M_{\odot}\right) \\
(2)\end{array}$ & $\begin{array}{c}\text { MSTG } \\
r_{c} \\
(\mathrm{kpc}) \\
(3)\end{array}$ & $\begin{array}{c}v_{0} \\
\left(\mathrm{~km} \mathrm{~s}^{-1}\right) \\
(4)\end{array}$ & $\begin{array}{c}M \\
\left(10^{10} M_{\odot}\right) \\
(5)\end{array}$ & $\begin{array}{c}\text { MOND } \\
r_{c} \\
(\mathrm{kpc}) \\
(6)\end{array}$ & $\begin{array}{c}\underset{v_{c}}{\longrightarrow} \\
\left(\mathrm{km} \mathrm{s}^{-1}\right) \\
(7)\end{array}$ \\
\hline F583-1 & $1.56 \pm 0.12$ & $1.28 \pm 0.06$ & $93.2 \pm 2.3$ & $0.90 \pm 0.06$ & $1.81 \pm 0.07$ & $109.4 \pm 1.9$ \\
\hline NGC 247 & $2.27 \pm 0.17$ & $1.11 \pm 0.06$ & $109.4 \pm 2.8$ & $1.46 \pm 0.14$ & $1.35 \pm 0.10$ & $123.7 \pm 2.9$ \\
\hline NGC 598 & $1.78 \pm 0.04$ & $0.64 \pm 0.01$ & $110.9 \pm 0.8$ & $1.15 \pm 0.02$ & $0.60 \pm 0.01$ & $116.4 \pm 0.5$ \\
\hline NGC 1003 & $1.64 \pm 0.03$ & $0.08 \pm 0.00$ & $121.5 \pm 0.8$ & $1.66 \pm 0.03$ & $0.08 \pm 0.00$ & $127.6 \pm 0.6$ \\
\hline NGC 1417 & $16.60 \pm 0.49$ & $0.92 \pm 0.02$ & $238.2 \pm 2.8$ & $23.30 \pm 0.84$ & $1.15 \pm 0.03$ & $247.0 \pm 2.2$ \\
\hline NGC 3495 & $4.16 \pm 0.27$ & $0.87 \pm 0.04$ & $142.1 \pm 3.3$ & $4.01 \pm 0.28$ & $0.95 \pm 0.04$ & $159.2 \pm 2.7$ \\
\hline NGC 3672 & $14.86 \pm 0.20$ & $1.21 \pm 0.01$ & $215.2 \pm 1.2$ & $17.02 \pm 0.25$ & $1.32 \pm 0.01$ & $228.4 \pm 0.8$ \\
\hline NGC 3917 & $6.25 \pm 0.45$ & $1.60 \pm 0.09$ & $142.8 \pm 3.8$ & $5.18 \pm 0.52$ & $1.89 \pm 0.15$ & $169.7 \pm 4.2$ \\
\hline NGC 4010 & $5.56 \pm 0.88$ & $1.62 \pm 0.17$ & $136.2 \pm 7.9$ & $4.66 \pm 0.87$ & $2.03 \pm 0.24$ & $165.2 \pm 7.7$ \\
\hline NGC 4183 & $2.04 \pm 0.11$ & $0.85 \pm 0.05$ & $111.3 \pm 2.0$ & $1.35 \pm 0.07$ & $0.91 \pm 0.06$ & $121.2 \pm 1.5$ \\
\hline UGC 6446 & $0.83 \pm 0.04$ & $0.73 \pm 0.04$ & $85.1 \pm 1.4$ & $0.43 \pm 0.02$ & $0.90 \pm 0.06$ & $91.2 \pm 1.3$ \\
\hline UGC 6614 & $11.36 \pm 1.79$ & $1.24 \pm 0.22$ & $192.3 \pm 11.9$ & $9.90 \pm 1.18$ & $1.14 \pm 0.16$ & $199.5 \pm 5.9$ \\
\hline UGC 6930 & $2.17 \pm 0.13$ & $1.03 \pm 0.06$ & $109.5 \pm 2.2$ & $1.40 \pm 0.07$ & $1.19 \pm 0.07$ & $122.2 \pm 1.6$ \\
\hline UGC 6983 & $2.12 \pm 0.16$ & $0.90 \pm 0.07$ & $111.5 \pm 2.8$ & $1.34 \pm 0.10$ & $0.95 \pm 0.09$ & $121.0 \pm 2.3$ \\
\hline \multicolumn{7}{|c|}{ HSB Galaxies } \\
\hline IC 342 & $7.95 \pm 0.14$ & $1.36 \pm 0.03$ & $188.3 \pm 1.2$ & $8.68 \pm 0.16$ & $1.44 \pm 0.03$ & $193.0 \pm 0.9$ \\
\hline Milky Way & $9.12 \pm 0.28$ & $1.04 \pm 0.05$ & $204.8 \pm 2.4$ & $10.60 \pm 0.37$ & $1.18 \pm 0.05$ & $202.9 \pm 1.8$ \\
\hline NGC 224 & $20.19 \pm 0.30$ & $1.84 \pm 0.04$ & $259.6 \pm 1.6$ & $25.54 \pm 0.45$ & $2.17 \pm 0.05$ & $252.8 \pm 1.1$ \\
\hline NGC 253 & $6.94 \pm 0.25$ & $0.86 \pm 0.04$ & $188.0 \pm 2.5$ & $7.88 \pm 0.31$ & $0.95 \pm 0.04$ & $188.4 \pm 1.9$ \\
\hline NGC 300 & $2.03 \pm 0.17$ & $2.70 \pm 0.19$ & $101.7 \pm 2.9$ & $1.03 \pm 0.09$ & $2.93 \pm 0.25$ & $113.3 \pm 2.5$ \\
\hline NGC 660 & $3.20 \pm 0.06$ & $0.54 \pm 0.02$ & $146.6 \pm 0.9$ & $2.99 \pm 0.06$ & $0.48 \pm 0.02$ & $147.8 \pm 0.7$ \\
\hline NGC 801 & $20.07 \pm 2.09$ & $2.65 \pm 0.24$ & $240.3 \pm 10.2$ & $17.90 \pm 2.05$ & $2.44 \pm 0.23$ & $231.3 \pm 6.6$ \\
\hline NGC 891 & $7.47 \pm 0.17$ & $0.78 \pm 0.03$ & $194.9 \pm 1.7$ & $8.49 \pm 0.24$ & $0.87 \pm 0.04$ & $192.0 \pm 1.3$ \\
\hline NGC 1068 & $9.42 \pm 0.54$ & $1.11 \pm 0.07$ & $205.9 \pm 4.5$ & $12.96 \pm 0.87$ & $1.46 \pm 0.09$ & $213.4 \pm 3.6$ \\
\hline NGC 1097 & $22.68 \pm 0.31$ & $1.19 \pm 0.03$ & $290.1 \pm 1.6$ & $29.64 \pm 0.51$ & $1.49 \pm 0.04$ & $262.4 \pm 1.1$ \\
\hline NGC 1365 & $14.96 \pm 0.25$ & $1.29 \pm 0.03$ & $242.6 \pm 1.6$ & $18.51 \pm 0.36$ & $1.52 \pm 0.04$ & $233.2 \pm 1.1$ \\
\hline NGC 1808 & $4.10 \pm 0.10$ & $0.51 \pm 0.02$ & $160.6 \pm 1.4$ & $4.32 \pm 0.12$ & $0.53 \pm 0.02$ & $162.1 \pm 1.1$ \\
\hline NGC 2403 & $3.80 \pm 0.13$ & $2.09 \pm 0.07$ & $133.7 \pm 1.6$ & $2.77 \pm 0.09$ & $1.95 \pm 0.07$ & $145.1 \pm 1.1$ \\
\hline NGC 2590 & $14.05 \pm 0.48$ & $1.10 \pm 0.05$ & $241.0 \pm 3.3$ & $17.54 \pm 0.71$ & $1.33 \pm 0.07$ & $230.1 \pm 2.3$ \\
\hline NGC 2841 & $33.04 \pm 1.31$ & $2.19 \pm 0.14$ & $308.3 \pm 5.2$ & $41.07 \pm 0.80$ & $2.46 \pm 0.07$ & $284.7 \pm 1.4$ \\
\hline NGC 2903 & $9.66 \pm 0.61$ & $1.72 \pm 0.11$ & $195.9 \pm 4.8$ & $10.61 \pm 0.75$ & $1.82 \pm 0.12$ & $202.9 \pm 3.6$ \\
\hline NGC 2998 & $15.13 \pm 1.20$ & $2.52 \pm 0.19$ & $216.7 \pm 6.8$ & $14.05 \pm 1.25$ & $2.39 \pm 0.19$ & $217.7 \pm 4.8$ \\
\hline
\end{tabular}


Table 3-Continued

\begin{tabular}{|c|c|c|c|c|c|c|}
\hline Galaxy & $\begin{array}{c}M \\
\left(10^{10} M_{\odot}\right) \\
(2)\end{array}$ & $\begin{array}{c}\text { MSTG } \\
r_{c} \\
(\mathrm{kpc}) \\
(3)\end{array}$ & $\begin{array}{c}\underset{v_{0}}{\longrightarrow} \\
\left(\mathrm{km} \mathrm{s}^{-1}\right) \\
(4)\end{array}$ & $\begin{array}{c}M \\
\left(10^{10} M_{\odot}\right) \\
(5)\end{array}$ & $\begin{array}{c}\text { MOND } \\
r_{c} \\
(\mathrm{kpc}) \\
(6)\end{array}$ & $\begin{array}{c}\underset{v_{c}}{\longrightarrow} \\
\left(\mathrm{km} \mathrm{s}^{-1}\right) \\
(7)\end{array}$ \\
\hline NGC 3031 & $6.95 \pm 0.12$ & $0.67 \pm 0.02$ & $191.8 \pm 1.3$ & $7.97 \pm 0.17$ & $0.75 \pm 0.02$ & $188.9 \pm 1.0$ \\
\hline NGC 3034 & $0.52 \pm 0.03$ & $0.08 \pm 0.01$ & $85.0 \pm 1.6$ & $0.59 \pm 0.05$ & $0.09 \pm 0.01$ & $98.4 \pm 2.0$ \\
\hline NGC 3079 & $8.73 \pm 0.23$ & $0.77 \pm 0.03$ & $207.1 \pm 2.1$ & $10.42 \pm 0.33$ & $0.91 \pm 0.04$ & $202.0 \pm 1.6$ \\
\hline NGC 3198 & $5.55 \pm 0.28$ & $2.18 \pm 0.12$ & $152.1 \pm 2.8$ & $4.49 \pm 0.26$ & $2.05 \pm 0.15$ & $163.7 \pm 2.4$ \\
\hline NGC 3379 & $6.99 \pm 0.06$ & $0.45 \pm 0.01$ & $196.7 \pm 0.6$ & $8.13 \pm 0.08$ & $0.51 \pm 0.01$ & $189.9 \pm 0.5$ \\
\hline NGC 3379 & $6.99 \pm 0.06$ & $0.45 \pm 0.01$ & $196.7 \pm 0.6$ & $8.13 \pm 0.08$ & $0.51 \pm 0.01$ & $189.9 \pm 0.5$ \\
\hline NGC 3521 & $7.89 \pm 0.10$ & $0.80 \pm 0.02$ & $198.7 \pm 1.0$ & $9.12 \pm 0.14$ & $0.89 \pm 0.02$ & $195.4 \pm 0.8$ \\
\hline NGC 3628 & $9.13 \pm 0.31$ & $1.17 \pm 0.05$ & $202.3 \pm 2.6$ & $10.67 \pm 0.41$ & $1.32 \pm 0.06$ & $203.2 \pm 2.0$ \\
\hline NGC 3726 & $9.60 \pm 1.37$ & $4.07 \pm 0.58$ & $158.4 \pm 8.8$ & $7.22 \pm 1.06$ & $4.00 \pm 0.72$ & $184.3 \pm 6.8$ \\
\hline NGC 3769 & $2.59 \pm 0.24$ & $1.66 \pm 0.20$ & $121.7 \pm 3.8$ & $1.61 \pm 0.16$ & $1.34 \pm 0.24$ & $126.6 \pm 3.2$ \\
\hline NGC 3893 & $7.70 \pm 1.00$ & $1.74 \pm 0.29$ & $179.3 \pm 8.9$ & $7.85 \pm 1.17$ & $1.78 \pm 0.34$ & $188.2 \pm 7.0$ \\
\hline NGC 3953 & $20.47 \pm 1.65$ & $3.46 \pm 0.28$ & $225.5 \pm 7.4$ & $24.30 \pm 1.94$ & $3.92 \pm 0.32$ & $249.7 \pm 5.0$ \\
\hline NGC 3992 & $25.16 \pm 2.32$ & $2.77 \pm 0.44$ & $260.9 \pm 10.0$ & $28.35 \pm 3.90$ & $2.74 \pm 0.72$ & $259.5 \pm 8.9$ \\
\hline NGC 4013 & $6.01 \pm 0.35$ & $0.70 \pm 0.19$ & $181.1 \pm 3.9$ & $5.52 \pm 0.26$ & $0.16 \pm 0.17$ & $172.4 \pm 2.1$ \\
\hline NGC 4051 & $7.21 \pm 1.31$ & $2.58 \pm 0.43$ & $161.7 \pm 11.1$ & $6.20 \pm 1.22$ & $2.50 \pm 0.47$ & $177.4 \pm 8.8$ \\
\hline NGC 4088 & $9.74 \pm 1.52$ & $3.15 \pm 0.51$ & $172.4 \pm 10.4$ & $8.87 \pm 1.46$ & $3.23 \pm 0.60$ & $194.0 \pm 8.0$ \\
\hline NGC 4100 & $10.30 \pm 1.59$ & $2.89 \pm 0.49$ & $180.2 \pm 10.8$ & $9.91 \pm 1.64$ & $3.00 \pm 0.58$ & $199.5 \pm 8.2$ \\
\hline NGC 4138 & $4.31 \pm 0.90$ & $0.68 \pm 0.39$ & $160.7 \pm 12.1$ & $4.25 \pm 1.03$ & $0.62 \pm 0.45$ & $161.4 \pm 9.8$ \\
\hline NGC 4157 & $11.64 \pm 1.21$ & $2.92 \pm 0.36$ & $188.5 \pm 7.7$ & $11.05 \pm 1.28$ & $2.93 \pm 0.43$ & $205.0 \pm 5.9$ \\
\hline NGC 4217 & $12.92 \pm 1.54$ & $3.31 \pm 0.36$ & $189.7 \pm 8.9$ & $13.01 \pm 1.66$ & $3.49 \pm 0.42$ & $213.6 \pm 6.8$ \\
\hline NGC 4258 & $7.29 \pm 0.14$ & $0.84 \pm 0.03$ & $191.9 \pm 1.4$ & $7.79 \pm 0.16$ & $0.86 \pm 0.03$ & $187.8 \pm 1.0$ \\
\hline NGC 4303 & $3.08 \pm 0.08$ & $0.59 \pm 0.02$ & $143.8 \pm 1.4$ & $2.92 \pm 0.08$ & $0.55 \pm 0.02$ & $146.9 \pm 1.0$ \\
\hline NGC 4321 & $21.67 \pm 0.45$ & $2.12 \pm 0.06$ & $260.2 \pm 2.2$ & $28.20 \pm 0.68$ & $2.56 \pm 0.07$ & $259.1 \pm 1.6$ \\
\hline NGC 4448 & $1.98 \pm 0.08$ & $0.27 \pm 0.01$ & $127.8 \pm 1.7$ & $2.10 \pm 0.09$ & $0.28 \pm 0.01$ & $135.4 \pm 1.4$ \\
\hline NGC 4527 & $5.55 \pm 0.23$ & $0.79 \pm 0.05$ & $174.3 \pm 2.7$ & $6.02 \pm 0.28$ & $0.84 \pm 0.05$ & $176.2 \pm 2.1$ \\
\hline NGC 4565 & $18.11 \pm 0.21$ & $1.72 \pm 0.03$ & $251.2 \pm 1.2$ & $21.74 \pm 0.32$ & $1.93 \pm 0.04$ & $242.8 \pm 0.9$ \\
\hline NGC 4631 & $6.15 \pm 0.10$ & $1.34 \pm 0.03$ & $171.4 \pm 1.0$ & $6.36 \pm 0.12$ & $1.37 \pm 0.03$ & $178.6 \pm 0.8$ \\
\hline NGC 4736 & $3.15 \pm 0.08$ & $0.47 \pm 0.02$ & $146.8 \pm 1.3$ & $3.21 \pm 0.09$ & $0.47 \pm 0.02$ & $150.5 \pm 1.1$ \\
\hline NGC 4945 & $4.58 \pm 0.12$ & $0.63 \pm 0.03$ & $165.1 \pm 1.6$ & $4.65 \pm 0.14$ & $0.63 \pm 0.03$ & $165.1 \pm 1.2$ \\
\hline NGC 5033 & $9.90 \pm 0.51$ & $1.10 \pm 0.08$ & $210.2 \pm 4.2$ & $10.80 \pm 0.70$ & $1.15 \pm 0.10$ & $203.9 \pm 3.3$ \\
\hline NGC 5055 & $8.38 \pm 0.06$ & $1.11 \pm 0.01$ & $196.9 \pm 0.5$ & $8.44 \pm 0.08$ & $1.07 \pm 0.02$ & $191.6 \pm 0.5$ \\
\hline NGC 5194 & $7.29 \pm 0.23$ & $0.61 \pm 0.03$ & $196.6 \pm 2.3$ & $8.72 \pm 0.30$ & $0.71 \pm 0.03$ & $193.2 \pm 1.7$ \\
\hline NGC 5236 & $6.16 \pm 0.12$ & $1.10 \pm 0.04$ & $175.5 \pm 1.3$ & $5.56 \pm 0.13$ & $0.96 \pm 0.04$ & $172.6 \pm 1.0$ \\
\hline NGC 5457 & $10.20 \pm 0.27$ & $1.39 \pm 0.04$ & $206.5 \pm 2.1$ & $12.03 \pm 0.36$ & $1.57 \pm 0.05$ & $209.4 \pm 1.6$ \\
\hline
\end{tabular}


Table 3-Continued

\begin{tabular}{|c|c|c|c|c|c|c|}
\hline Galaxy & $\begin{array}{c}\longleftarrow \\
\left(10^{10} M_{\odot}\right) \\
(2)\end{array}$ & $\begin{array}{c}\text { MSTG } \\
r_{c} \\
(\mathrm{kpc}) \\
(3)\end{array}$ & $\begin{array}{c}\longrightarrow \\
v_{0} \\
\left(\mathrm{~km} \mathrm{~s}^{-1}\right) \\
(4)\end{array}$ & $\begin{array}{c}\frac{4}{M} \\
\left(10^{10} M_{\odot}\right)\end{array}$ & $\begin{array}{c}\text { MOND } \\
r_{c} \\
(\mathrm{kpc}) \\
(6)\end{array}$ & $\begin{array}{c}\longrightarrow \\
\left.\left(\mathrm{km} \mathrm{s}_{c}\right)^{-1}\right) \\
(7)\end{array}$ \\
\hline NGC 5533 & $28.81 \pm 1.92$ & $2.11 \pm 0.23$ & $293.2 \pm 8.2$ & $25.61 \pm 1.68$ & $1.68 \pm 0.18$ & $253.0 \pm 4.1$ \\
\hline NGC 5907 & $4.59 \pm 0.26$ & $0.40 \pm 0.05$ & $169.3 \pm 3.5$ & $23.16 \pm 0.46$ & $2.35 \pm 0.06$ & $246.7 \pm 1.2$ \\
\hline NGC 6503 & $1.98 \pm 0.06$ & $1.10 \pm 0.05$ & $117.4 \pm 1.3$ & $1.38 \pm 0.05$ & $0.91 \pm 0.05$ & $122.0 \pm 1.0$ \\
\hline NGC 6674 & $32.48 \pm 2.38$ & $3.27 \pm 0.33$ & $277.7 \pm 8.6$ & $28.04 \pm 2.43$ & $2.70 \pm 0.33$ & $258.7 \pm 5.6$ \\
\hline NGC 6946 & $8.95 \pm 0.65$ & $3.54 \pm 0.27$ & $161.2 \pm 4.5$ & $7.29 \pm 0.61$ & $3.57 \pm 0.37$ & $184.8 \pm 3.8$ \\
\hline NGC 6951 & $6.22 \pm 0.22$ & $0.58 \pm 0.03$ & $185.8 \pm 2.5$ & $6.92 \pm 0.28$ & $0.63 \pm 0.03$ & $182.4 \pm 1.9$ \\
\hline NGC 7331 & $21.47 \pm 0.76$ & $2.56 \pm 0.10$ & $248.9 \pm 3.6$ & $24.73 \pm 0.83$ & $2.74 \pm 0.10$ & $250.8 \pm 2.1$ \\
\hline UGC 6973 & $6.41 \pm 0.45$ & $1.43 \pm 0.12$ & $172.5 \pm 4.5$ & $6.63 \pm 0.46$ & $1.46 \pm 0.12$ & $180.4 \pm 3.1$ \\
\hline
\end{tabular}

Note. - Best fit results according to both MSTG and MOND via a parametric mass distribution (independent of luminosity observations) - corresponding to the galaxy rotation curves of Figs. 24. Column (1) is the NGC/UGC galaxy number. The MSTG best fit results are presented in Columns (2) - (4), where Column (2) is the MSTG predicted total mass of the galaxy, $M$; Column (3) is the MSTG predicted core radius, $r_{c}$; and Column (4) is the predicted MSTG flat rotation velocity, $v_{0}$, of equation (35). The MOND best results are presented in Columns (5) - (7), where Column (5) is the MOND predicted mass of the galaxy, $M$; Column (6) is the MOND predicted core radius, $r_{c}$; and Column (7) is the MOND asymptotic velocity, $v_{c}$ of equation (31).

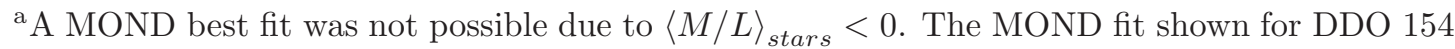
neglects $M_{\text {disk }}$, whereas all the data was used for the MSTG result. 
Table 4. Observed \& Actual Tully-Fisher Relation Results

\begin{tabular}{|c|c|c|c|c|}
\hline & $\begin{array}{c}B \text {-band } \\
\text { (1) }\end{array}$ & $\begin{array}{c}K \text {-band } \\
(2)\end{array}$ & $\begin{array}{c}\text { MSTG } \\
(3)\end{array}$ & $\begin{array}{c}\text { MOND } \\
(4)\end{array}$ \\
\hline \multicolumn{5}{|c|}{ UMa Cluster of Galaxies — Photometric Fits } \\
\hline$a$ & $2.67 \pm 0.25$ & $4.73 \pm 0.44$ & $3.19 \pm 0.10$ & $4.00 \pm 0.10$ \\
\hline$b$ & $-5.73 \pm 0.54$ & $-9.82 \pm 0.95$ & $-6.54 \pm 0.21$ & $-8.20 \pm 0.01$ \\
\hline \multicolumn{5}{|c|}{ Complete Sample of Galaxies — Parametric Fits } \\
\hline$a$ & $2.89 \pm 0.22$ & $2.85 \pm 0.33$ & $2.68 \pm 0.07$ & $4.00 \pm 0.00$ \\
\hline$b$ & $-6.30 \pm 0.47$ & $-5.73 \pm 0.74$ & $-5.18 \pm 0.16$ & $-8.21 \pm 0.00$ \\
\hline
\end{tabular}

Note. — The $B$-band luminosity data are taken from the original references except for Sofue (1996) and Romanowsky et al. (2003) which are taken from Tully (1988). The K-band luminosity data are taken from the 2MASS except for the Schombert F-type galaxies, which are taken from the original reference. The values of $a$ and $b$ are determined using a nonlinear least-squares fitting routine including estimated errors. Columns (1) and (2) are the best fit solutions to the observed Tully-Fisher relation, $\log (L)=a \log \left(v_{\text {out }}\right)+b$ in the $B$ - and $K$-bands, respectively. Columns (3) and (4) are the best fit solutions to the actual TullyFisher relation, $\log (M)=a \log (v)+b$ for MSTG and MOND, where $\mathrm{v}$ is determined by equations (41) and (42), respectively. The observed \& actual Tully-Fisher relation presented here are shown graphically in Figs. 6 \& 7, respectively. 